\title{
THE CONVEXITY PACKAGE FOR HAMILTONIAN ACTIONS ON CONFORMAL SYMPLECTIC MANIFOLDS
}

\author{
YOUMING CHEN, REYER SJAMAAR, AND XIANGDONG YANG
}

\begin{abstract}
Consider a Hamiltonian action of a compact connected Lie group on a conformal symplectic manifold. We prove a convexity theorem for the moment map under the assumption that the action is of Lee type, which establishes an analog of Kirwan's convexity theorem in conformal symplectic geometry.
\end{abstract}

\section{Contents}

1. Introduction

2. Preliminaries

3. Hamiltonian actions and the local model

4. Convexity properties of the moment map

5. Symplectic, conformal symplectic, and presymplectic convexity

6. Convexity for conformal symplectic manifolds of the first kind

7. Examples

Appendix A. An instance of real symplectic convexity

Appendix B. Conformal presymplectic convexity 28

References

\section{INTRODUCTION}

One of the most interesting results concerning the geometry of Hamiltonian actions of compact Lie groups on symplectic manifolds is the convexity theorem for the moment map image. An early instance of this theorem is Kostant's result [21] of 1973 for torus actions on conjugacy classes and flag manifolds. In 1982, Atiyah [1] and Guillemin-Sternberg [15] independently proved the convexity theorem in the case of torus actions. For an action of a non-abelian Lie group the moment map image is in general not convex. However, Guillemin-Sternberg [15] showed that if $M$ is an integral Kähler manifold the quotient space of the moment image by the coadjoint $K$-action is convex and they conjectured the same result for general symplectic

Date: June 4, 2019.

2010 Mathematics Subject Classification. 53D20; 58D19.

Key words and phrases. Conformal symplectic manifolds; moment maps; convexity properties.

This work was partly supported by the National Natural Science Foundation of China (grants No. 11571242 and No. 11701051) and the China Scholarship Council. 
manifolds. Subsequently, Kirwan [20] confirmed Guillemin and Sternberg's conjecture in 1984. The symplectic convexity theorem has versions for many other geometric objects, such as symplectic orbifolds, contact manifolds, generalized complex manifolds, presymplectic manifolds, and so on (see e.g. [27, 25, 32, 7, 35, 30, 14]).

From a conformal point of view, the closest geometric structure to a symplectic structure is a (locally) conformal(ly) symplectic structure. This type of structure was first introduced by Lee [23] and studied by Libermann [28], Lefebvre [24], GrayHervella [11], Vaisman [38], Eliashberg-Murphy [9], and Chantraine-Murphy [6]. (The term "locally conformally symplectic" is current in much of the literature, but for the sake of brevity we will follow [9] and say "conformal symplectic".) Conformal symplectic manifolds serve as natural phase spaces for certain problems in mechanics, such as Gaussian isokinetic dynamics and Nosé-Hoovers dynamics (cf. [39]). Conformal symplectic geometry relates closely to several other geometries. For instance, it is known that many non-Kähler compact complex surfaces admit locally conformal Kähler forms; see [4,33] and the references therein. Conformal symplectic manifolds are nothing but even-dimensional transitive Jacobi manifolds; see [12]. A conformal symplectic manifold of the first kind is a special example of a contact pair and therefore possesses an intrinsic transversely symplectic foliation; see [2]. Thus the geometry of conformal symplectic manifolds of the first kind is a special type of transversely symplectic geometry.

Belgun-Goertsches-Petrecca [5] have recently extended the convexity theorem of Atiyah and Guillemin-Sternberg to Hamiltonian torus actions on conformal symplectic manifolds. The purpose of this paper is to generalize their result to the nonabelian case.

1.1. Theorem. Let $K$ be a compact connected Lie group which acts on a connected strict conformal symplectic manifold $(M, \omega, \theta)$ in a Hamiltonian fashion. Assume that the $K$-action is of Lee type and that the moment map $\Phi: M \rightarrow \mathfrak{k}^{*}$ is proper, where $\mathfrak{k}=\operatorname{Lie}(K)$. Choose a maximal torus $T$ of $K$ and a closed Weyl chamber $C$ in $\mathfrak{t}^{*}$, where $\mathfrak{t}=\operatorname{Lie}(T)$.

(i) The fibres of $\Phi$ are connected and $\Phi: M \rightarrow \Phi(M)$ is an open map.

(ii) $\Delta(M)=\Phi(M) \cap C$ is a closed convex polyhedral set.

The proof this theorem, which is a variation on the argument of [5], is contained in Section 4. We also state a version for global conformal symplectic manifolds, Theorem 4.7, and a version for conformal presymplectic manifolds, Theorem B.1, in Appendix B. In Section 5 we investigate the relation between the moment map image of $M$ and that of its symplectic covering manifolds $\tilde{M}$, and obtain Theorems 5.2 and 5.4, which also generalize results for torus actions obtained in [5]. In addition we introduce a foliation of $M$ into presymplectic manifolds, which we call the Lee foliation, and we prove a stability result, Theorem 5.7, which combines a form of Reeb stability with a stability statement for the moment map images of the leaves of the Lee foliation. In Section 6, we present an alternative proof of the convexity theorem for conformal symplectic manifolds of the first kind from the viewpoint of transverse symplectic geometry (Theorem 6.5). The properness hypothesis on the moment map in Theorem 1.1 must not be taken too seriously. It is violated in some cases of interest where the conclusion of the theorem nevertheless holds. A few such examples are discussed in Section 7 and Appendix A. 
Acknowledgements. Youming Chen and Xiangdong Yang would like to thank the Departments of Mathematics at Pennsylvania State University and Cornell University, respectively, for their hospitality and their excellent working environment. They would like to express their great gratitude to the China Scholarship Council for financially supporting their visits. Reyer Sjamaar and Xiangdong Yang are grateful to Yi Lin for many fruitful discussions.

\section{Preliminaries}

In this section we establish some notational conventions and present a brief review of conformal symplectic structures.

Notation and conventions. Throughout this paper $K$ will denote a compact connected Lie group with Lie algebra $\mathfrak{k}$ and $M$ will denote a paracompact smooth manifold on which $K$ acts smoothly. We denote by $\mathfrak{X}(M)$ the Lie algebra of smooth vector fields on $M$. The stabilizer of a point $m \in M$ is denoted by $K_{m}$ and the orbit through $m$ by $K \cdot m$. The vector field on $M$ generated by the infinitesimal action of $\xi \in \mathfrak{k}$ is denoted by $\xi_{M}$. A differential form $\alpha \in \Omega^{p}(M)$ is horizontal if $\iota\left(\xi_{M}\right) \alpha=0$ for all $\xi \in \mathfrak{k}$. A $K$-invariant horizontal form $\alpha$ is said to be basic with respect to the $K$-action. We denote by $\Omega_{K \text {,bas }}^{p}(M)$ the space of basic $p$-forms. Since the exterior differential operator $d$ preserves the basic forms we get a complex $\left(\Omega_{K, \text { bas }}^{*}(M), d\right)$. The associated cohomology, denoted by $H_{K \text {,bas }}^{*}(M)$, is called the basic cohomology of the $K$-manifold $M$.

We denote by $\mathbb{R}_{>0}$ the multiplicative group of positive real numbers and by $\mathcal{C}^{\infty}\left(M, \mathbb{R}_{>0}\right)$ the group of smooth positive-valued functions on $M$.

Let $V$ be a finite-dimensional vector space over $\mathbb{R}$. A closed affine halfspace is a subset of $V$ defined by an inequality $\langle\phi, v\rangle \geq c$ with $\phi \in V^{*}$ and $c \in \mathbb{R}$. A convex polyhedral set is the intersection of a locally finite collection of closed affine halfspaces in $V$. A convex polyhedron is the intersection of finitely many closed affine halfspaces. A convex polytope is a bounded convex polyhedron. Suppose $V$ is defined over $\mathbb{Q}$. A closed affine halfspace is rational (resp. semirational) if it is defined by an inequality $\langle\phi, v\rangle \geq c$ with $\phi \in V_{\mathbb{Q}}^{*}$ and $c \in \mathbb{Q}$ (resp. $\phi \in V_{\mathbb{Q}}^{*}$ and $c \in \mathbb{R}$ ). A rational (resp. semirational) convex polyhedral set is the intersection of a locally finite collection of rational (resp. semirational) closed affine halfspaces in $V$. Thus a semirational convex polyhedral set has a rational normal vector at each facet, but its vertices may not be rational.

Conformal symplectic structures. Let $M$ be a smooth manifold. An almost symplectic form is a non-degenerate 2 -form on $M$. An almost symplectic form $\omega$ is conformal symplectic if there exist an open covering $\left\{U_{\lambda}\right\}_{\lambda \in \Lambda}$ of $M$ and a family of smooth functions $\left\{f_{\lambda}: U_{\lambda} \rightarrow \mathbb{R}\right\}$ such that $\exp \left(f_{\lambda}\right) \cdot\left(\left.\omega\right|_{U_{\lambda}}\right)$ is symplectic. The nondegeneracy of $\omega$ implies $d f_{\lambda}=d f_{\mu}$ on $U_{\lambda} \cap U_{\mu}$. Consequently, the forms $\left\{d f_{\lambda}\right\}$ glue to a globally defined closed 1-form $\theta$, which satisfies $d \omega=-\theta \wedge \omega$. If $\operatorname{dim} M \geq 4$, the form $\theta$ is uniquely determined by $\omega$.

2.1. Definition. A conformal symplectic structure on $M$ is a pair $(\omega, \theta)$, where $\omega$ is an almost symplectic form and $\theta$ a closed 1-form with the property that $d \omega+\theta \wedge \omega=$ 0 . The form $\theta$ is the Lee form and its cohomology class $[\theta] \in H^{1}(M ; \mathbb{R})$ is the Lee class of the conformal symplectic structure. A conformal symplectic structure $(\omega, \theta)$ is strict if $\theta$ is not exact, and global if $\theta$ is exact. A conformal symplectic manifold is a triple $(M, \omega, \theta)$, where $(\omega, \theta)$ is a conformal symplectic structure on $M$. 
The Lee form associated with a conformal symplectic structure defines a flat connection on the trivial line bundle $M \times \mathbb{R}$, or equivalently on the trivial principal bundle $M \times \mathbb{R}_{>0}$. The group of gauge transformations of this principal bundle is the group $\mathcal{C}^{\infty}\left(M, \mathbb{R}_{>0}\right)$ of smooth positive-valued functions. The gauge action of a function $a>0$ on a conformal symplectic structure $(\omega, \theta)$ is given by

$$
a \cdot(\omega, \theta)=(a \omega, \theta-d \log a) .
$$

Conformal symplectic structures related by the gauge action are called conformally equivalent. A conformal symplectic structure $(\omega, \theta)$ is global if and only if it is conformally equivalent to a symplectic structure, namely $e^{f} \cdot(\omega, \theta)=\left(e^{f} \omega, 0\right)$ if $\theta=d f$.

It is known that every compact almost symplectic manifold $\left(M, \omega_{0}\right)$ with nonzero cohomology group $H^{1}(M ; \mathbb{Z})$ admits a conformal symplectic structure. More precisely, the $h$-principle for conformal symplectic manifolds of Eliashberg-Murphy [9, Theorem 1.8] states that for each nonzero class $\Theta \in H^{1}(M ; \mathbb{Z})$ and for all sufficiently large $c>0$ there exists a conformal symplectic form $\omega$ which is homotopic to $\omega_{0}$ through almost symplectic forms and has Lee class equal to $[\theta]=c \Theta$.

Let $(M, \omega, \theta)$ be a conformal symplectic manifold. The connection $\theta$ defines a covariant derivative, also called the twisted differential, $d_{\theta}: \Omega^{*}(M) \rightarrow \Omega^{*+1}(M)$ given by

$$
d_{\theta} \alpha=d \alpha+\theta \wedge \alpha
$$

By definition $\omega$ and $\theta$ are $d_{\theta}$-closed. For a vector field $X \in \mathfrak{X}(M)$ the twisted Lie derivative $L_{\theta}(X): \Omega^{*}(M) \rightarrow \Omega^{*}(M)$ is the operator given by

$$
L_{\theta}(X) \alpha=L(X) \alpha+\theta(X) \alpha .
$$

These operators satisfy the usual rules of the Cartan differential calculus, namely

$$
\begin{aligned}
{[\iota(X), \iota(Y)] } & =0, & {\left[L_{\theta}(X), L_{\theta}(Y)\right] } & =L_{\theta}([X, Y]), \\
{\left[L_{\theta}(X), d_{\theta}\right] } & =0, & {\left[L_{\theta}(X), \iota(Y)\right] } & =\iota([X, Y]), \\
{\left[d_{\theta}, d_{\theta}\right] } & =0, & {\left[\iota(X), d_{\theta}\right] } & =L_{\theta}(X)
\end{aligned}
$$

for all $X, Y \in \mathfrak{X}(M)$, where the square brackets denote graded commutators. In particular, $\left(\Omega^{*}(M), d_{\theta}\right)$ is a cochain complex, the cohomology of which we denote by $H_{\theta}^{*}(M)$. The Lie algebra of vector fields $\mathfrak{X}(M)$ has several subspaces of interest, including

$$
\begin{aligned}
\mathfrak{X}_{\omega}(M) & =\{X \in \mathfrak{X}(M) \mid L(X) \omega=L(X) \theta=0\}, \\
\mathfrak{X}_{(\omega, \theta)}(M) & =\left\{X \in \mathfrak{X}(M) \mid L_{\theta}(X) \omega=0\right\}, \\
\mathfrak{X}_{[\omega]}(M) & =\left\{X \in \mathfrak{X}(M) \mid L(X) \omega=f \omega, L(X) \theta=-d f \text { for some } f \in \mathcal{C}^{\infty}(M)\right\} .
\end{aligned}
$$

Clearly, $\mathfrak{X}_{\omega}(M)$ and $\mathfrak{X}_{[\omega]}(M)$ are Lie subalgebras, and it follows from (2.2) that $\mathfrak{X}_{(\omega, \theta)}(M)$ is a Lie subalgebra as well. Plainly we have the inclusions

$$
\mathfrak{X}_{\omega}(M) \subseteq \mathfrak{X}_{[\omega]}(M), \quad \mathfrak{X}_{(\omega, \theta)}(M) \subseteq \mathfrak{X}_{[\omega]}(M) .
$$

The condition $L(X) \theta=0$ in the definition of $\mathfrak{X}_{\omega}(M)$ is usually superfluous. Namely if $L(X) \omega=0$, then

$$
0=d L(X) \omega=L(X) d \omega=-L(X) \theta \wedge \omega,
$$

so $L(X) \theta=0$ and $X \in \mathfrak{X}_{\omega}(M)$, provided that $\operatorname{dim}(X) \geq 4$. Similarly, the condition $L(X) \theta=-d f$ in the definition of $\mathfrak{X}_{[\omega]}(M)$ is superfluous if $\operatorname{dim}(X) \geq 4$. 
The (twisted) Hamiltonian vector field of a function $f \in \mathcal{C}^{\infty}(M)$ is the vector field $X_{f}$ defined by $\iota\left(X_{f}\right) \omega=d_{\theta} f$. In conformal symplectic geometry Hamiltonian vector fields do in general not preserve the conformal symplectic form. Instead we have $L_{\theta}\left(X_{f}\right) \omega=d_{\theta}^{2} f=0$, so $X_{f} \in \mathfrak{X}_{(\omega, \theta)}(M)$. In other words, the map $f \mapsto X_{f}$ is a map

$$
\mathcal{C}^{\infty}(M) \longrightarrow \mathfrak{X}_{(\omega, \theta)}(M) .
$$

An important role is played by the Hamiltonian vector field of the constant function 1 , which we will call the (symplectic) Lee vector field and denote by $A=X_{1}$. It is easy to show that

$$
\iota(A) \theta=0, \quad L(A) \theta=0, \quad L(A) \omega=0 .
$$

As in symplectic geometry, the bracket of two functions $f, g \in \mathcal{C}^{\infty}(M)$ defined by

$$
\{f, g\}=\omega\left(X_{f}, X_{g}\right)
$$

makes $\mathcal{C}^{\infty}(M)$ a Lie algebra, and the map (2.3) is a Lie algebra homomorphism, i.e. $X_{\{f, g\}}=\left[X_{f}, X_{g}\right]$. We will repeatedly use the following elementary result proved by Vaisman.

2.4. Proposition ([38, Proposition 2.1]). Let $(M, \omega, \theta)$ be a connected strict conformal symplectic manifold. Then $d_{\theta}: \mathcal{C}^{\infty}(M) \rightarrow \Omega^{1}(M)$ is injective. Hence the homomorphism (2.3) is injective and $H_{\theta}^{0}(M)=0$.

In contrast to the symplectic case the bracket $\{\cdot, \cdot\}$ does not satisfy the Leibniz identity and so is not a Poisson bracket (cf. [38, Section 2]). The deficiency of the Leibniz identity is also manifested in the fact that $d_{\theta}$ is not a derivation. In fact, we have $[\Pi, \Pi]=2 A \wedge \Pi$, where $\Pi$ is the 2 -vector field dual to $\omega$, and so the pair $(\Pi, A)$ defines a Jacobi structure on $M$ in the sense of Lichnerowicz [29]. It was proved in [12] (see also $[8, \S 3]$ ) that conformal symplectic structures are precisely the even-dimensional transitive Jacobi structures, whereas contact forms are the odd-dimensional transitive Jacobi structures.

\section{Hamiltonian ACTIONS AND THE LOCAL MODEL}

In this section $(M, \omega, \theta)$ denotes a connected conformal symplectic manifold on which the compact connected Lie group $K$ acts smoothly. We introduce Hamiltonian Lie group actions and actions of Lee type following [16] and [5] (with some minor changes in terminology) and prove a "local normal form" theorem for the moment map, which is one of the ingredients in the proof of the convexity theorem.

3.1. Definition. The $K$-action on $M$ is weakly Hamiltonian if there exists a map $\Phi: M \rightarrow \mathfrak{k}^{*}$, called a moment map, such that $\iota\left(\xi_{M}\right) \omega=d_{\theta} \Phi^{\xi}$ for all $\xi \in \mathfrak{k}$. Here $\Phi^{\xi}$ is defined by $\langle\Phi(x), \xi\rangle=\Phi^{\xi}(x)$. The $K$-action is Hamiltonian, or $M$ is a Hamiltonian conformal symplectic $K$-manifold, if there exists a moment map $\Phi$ which is $K$ equivariant with respect to the given action on $M$ and the coadjoint action on $\mathfrak{k}^{*}$, i.e. $\Phi(g \cdot x)=\operatorname{Ad}_{g^{-1}}^{*}(\Phi(x))$ for all $g \in K$.

If $\Phi$ is a moment map for the $K$-action on $M$, then $\xi_{M}=X_{\Phi^{\xi}}$, and hence $\xi_{M} \in \mathfrak{X}_{(\omega, \theta)}(M)$, for all $\xi \in \mathfrak{k}$. Define $\Phi^{\vee}: \mathfrak{k} \rightarrow \mathcal{C}^{\infty}(M)$ by $\Phi^{\vee}(\xi)=\Phi^{\xi}$. The map 
$\Phi^{\vee}$ is a lifting of the infinitesimal action $\mathfrak{k} \rightarrow \mathfrak{X}(M)$ to a linear map $\mathfrak{k} \rightarrow \mathcal{C}^{\infty}(M)$ along the map (2.3),

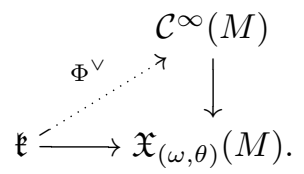

The action is Hamiltonian if and only if such a lifting exists. It follows from Proposition 2.4 that if $(M, \omega, \theta)$ is strict (which is the case of most interest to us), then the moment map $\Phi$ is uniquely determined by the $K$-action. For Hamiltonian $K$-actions on symplectic manifolds the moment map is unique up to an additive constant, and it is well-known that the constant can be chosen so that the moment map is equivariant with respect to the coadjoint action on $\mathfrak{k}^{*}$. However, in the conformal symplectic setting the moment map is not necessarily $K$-equivariant. The following proposition, which is due to [16] and [5], shows that the failure of the $K$-equivariance lies in the fact that Hamiltonian vector fields do not preserve the conformal symplectic form. We review the argument, because we will need a generalization of the statement to degenerate 2-forms in Appendix B.

3.2. Proposition ([16, Proposition 2], [5, Proposition 4.6]). Suppose that $(M, \omega, \theta)$ is strict conformal symplectic and that the $K$-action is weakly Hamiltonian with moment map $\Phi$. Then the following conditions are equivalent:

(i) The action is Hamiltonian.

(ii) $\omega$ is $K$-invariant.

(iii) The Lee form $\theta$ is $K$-basic.

Proof. The vector field $\xi_{M}$ is Hamiltonian, so $L_{\theta}\left(\xi_{M}\right) \omega=0$ for all $\xi \in \mathfrak{k}$, i.e. $L\left(\xi_{M}\right) \omega=\gamma_{\xi} \omega$, where $\gamma_{\xi}=-\theta\left(\xi_{M}\right) \in \mathcal{C}^{\infty}(M)$. It follows that $\omega$ is conformally $K$-invariant in the sense that $g^{*} \omega=c_{g} \omega$ for all $g \in K$. Here $c_{g} \in \mathcal{C}^{\infty}\left(M, \mathbb{R}_{>0}\right)$ is defined by

$$
c_{g}(x)=\exp \int_{0}^{1} \gamma_{\xi}\left(\exp _{K}\left(t \xi_{M}\right) \cdot x\right) d t
$$

for $x \in M$ and for any $\xi \in \mathfrak{k}$ with $g=\exp _{K}(\xi)$. Since $H_{\theta}^{0}(M)=0$ by Proposition 2.4, we have

$$
H^{1}\left(\mathfrak{k}, H_{\theta}^{0}(M)\right)=H^{2}\left(\mathfrak{k}, H_{\theta}^{0}(M)\right)=0,
$$

so it follows from [16, Proposition 3] that the moment map $\Phi$ is unique and has the conformal equivariance property $\Phi(g \cdot x)=c_{g}(x) \operatorname{Ad}_{g^{-1}}^{*}(\Phi(x))$ for all $g \in K$. The equivalence (i) $\Longleftrightarrow$ (ii) is now immediate, because $\omega$ is $K$-invariant if and only if $c_{g}$ is constant equal to 1 for all $g$. Also $\omega$ is invariant if and only if $L\left(\xi_{M}\right) \omega=0$, i.e. $\gamma_{\xi}=0$ for all $\xi$, so (ii) is equivalent to $\theta$ being horizontal. Every closed horizontal form is invariant, so $\theta$ is horizontal if and only if it is basic.

3.3. Remark. For Hamiltonian actions on symplectic manifolds, it is well-known that each component of the moment map is a Morse-Bott function. However, this fails in general for Hamiltonian actions on conformal symplectic manifolds.

Let $m \in M$. Recall that the orbit $K \cdot m$ has an equivariant tubular neighborhood, i.e. an open neighborhood of the form $V=K \cdot S$, where $S$ is a slice at $m$ for the $K$-action. A useful consequence of Proposition 3.2 is that such a neighborhood $V$ is conformally equivalent to a symplectic Hamiltonian $K$-manifold. 
3.4. Proposition. Suppose that $(M, \omega, \theta)$ is strict conformal symplectic and that the $K$-action is Hamiltonian with moment map $\Phi$. Let $m \in M$ and let $V$ be an equivariant tubular neighborhood of $K \cdot m$.

(i) There exists a $K$-invariant smooth function $f$ on $V$ such that $\left.\theta\right|_{V}=d f$.

(ii) The form $\Omega=\left.e^{f} \cdot \omega\right|_{V}$ is symplectic and the $K$-action on $(V, \Omega)$ is Hamiltonian with equivariant moment map $\Psi=\left.e^{f} \cdot \Phi\right|_{V}$.

Proof. (i) By Proposition 3.2 the form $\left.\theta\right|_{V} \in \Omega^{1}(V)$ is basic. A theorem of Koszul (see [22, p. 141] or [13, Appendix B3]) says that the basic de Rham complex $\Omega_{K \text {,bas }}^{*}(V)$ is acyclic. Hence $\left.\theta\right|_{V}=d f$ for some $K$-invariant $f \in \mathcal{C}^{\infty}(V)$.

(ii) The 2 -form $\left.\omega\right|_{V}$ is global conformal symplectic with Lee form $d f$. It follows that $\Omega=\left.e^{f} \cdot \omega\right|_{V}$ is a symplectic form. Since $f$ is $K$-invariant, the form $\Omega$ is $K$-invariant and the map $\Psi$ is $K$-equivariant. For all $\xi \in \mathfrak{k}$ we have

$$
d \Psi^{\xi}=d\left(\left.e^{f} \Phi^{\xi}\right|_{V}\right)=\left.e^{f}\left(d \Phi^{\xi}+\theta \cdot \Phi^{\xi}\right)\right|_{V}=\left.e^{f} d_{\theta} \Phi^{\xi}\right|_{V}=\left.e^{f} \iota\left(\xi_{V}\right) \omega\right|_{V}=\iota\left(\xi_{V}\right) \Omega,
$$

which shows that $\Psi$ is a moment map for the $K$-action on $(V, \Omega)$.

3.5. Definition. The $K$-action on $M$ is of Lee type if the Lee vector field $A$ is generated by the infinitesimal action of a Lie algebra element, i.e. $A=\zeta_{M}$ for some $\zeta \in \mathfrak{k}$. Such an element $\zeta$ is a Lee element for the $K$-action.

The Lee type condition is equivalent to the moment map image being contained in an affine hyperplane.

3.6. Lemma. Let $(M, \omega, \theta)$ be strict conformal symplectic. The $K$-action on $M$ is of Lee type, with Lee element $\zeta \in \mathfrak{k}$, if and only if the image of $\Phi$ lies in the affine hyperplane

$$
\mathcal{H}_{\zeta}=\left\{v \in \mathfrak{k}^{*} \mid\langle v, \zeta\rangle=1\right\} .
$$

Proof. By definition $d_{\theta} \Phi^{\zeta}=\iota\left(\zeta_{M}\right) \omega$. If $\Phi(M) \subseteq \mathcal{H}_{\zeta}$, then $\Phi^{\zeta}=1$, so $\theta=d_{\theta} 1=$ $d_{\theta} \Phi^{\zeta}=\iota\left(\zeta_{M}\right) \omega$. This shows that $A=\zeta_{M}$, i.e. the action is of Lee type with Lee element $\zeta$. Conversely, if $\zeta$ is a Lee element, then $d_{\theta} \Phi^{\zeta}=\theta=d_{\theta}(1)$. By Proposition 2.4, since $M$ is connected and $(\omega, \theta)$ is strict, the map $d_{\theta}: \mathcal{C}^{\infty}(M) \rightarrow$ $\Omega^{1}(M)$ is injective and therefore we get $\Phi^{\zeta}=1$, i.e. $\Phi(M) \subset \mathcal{H}_{\zeta}$.

The next result is a version of [5, Corollary 4.14].

3.7. Lemma. Let $(M, \omega, \theta)$ be strict conformal symplectic. Suppose that the $K$ action is weakly Hamiltonian and of Lee type. Then the K-action is Hamiltonian if and only if there exists a central Lee element for the $K$-action. In particular, the action is Hamiltonian if $K$ is a torus, and cannot be Hamiltonian if $K$ is semisimple.

Proof. Suppose the action is Hamiltonian. Then $\omega$ and $\theta$ are $K$-invariant by Proposition 3.2, so the Lee vector field $A$, which is characterized by $\iota(A) \omega=\theta$, is $K$-invariant. Choose any $\xi \in \mathfrak{k}$ such that $A=\xi_{M}$. Invariance of $A$ yields $\left(\operatorname{Ad}_{g} \xi\right)_{M}=g_{*}\left(\xi_{M}\right)=g_{*} A=A$ for all $g \in K$. Let $\zeta=\int_{K} \operatorname{Ad}_{g} \xi d g$, where $d g$ denotes normalized Haar measure on $K$. Then $\zeta$ is central and

$$
\zeta_{M}=\int_{K}\left(\operatorname{Ad}_{g} \xi\right)_{M} d g=\int_{K} A d g=A,
$$


so $\zeta$ is a Lee element. Conversely, suppose there exists a central Lee element $\zeta$. Then for all $\xi \in \mathfrak{g}$ we have

$$
\begin{aligned}
d_{\theta} \iota\left(\xi_{M}\right) \theta & =L_{\theta}\left(\xi_{M}\right) \theta \\
& =L_{\theta}\left(\xi_{M}\right) \iota\left(\zeta_{M}\right) \omega \\
& =\left[L_{\theta}\left(\xi_{M}\right), \iota\left(\zeta_{M}\right)\right] \omega-\iota\left(\zeta_{M}\right) L_{\theta}\left(\xi_{M}\right) \omega \\
& \left.=\iota\left([\xi, \zeta]_{M}\right]\right) \omega \\
& =0 .
\end{aligned}
$$

Here we used the commutation relations (2.2), the fact that $L_{\theta}\left(\xi_{M}\right) \omega=0$, which follows from the assumption that the vector field $\xi_{M}$ is Hamiltonian, and the fact that $\zeta$ is central. By Proposition 2.4 we conclude that $\iota\left(\xi_{M}\right) \theta=0$, i.e. $\theta$ is basic. Hence the action is Hamiltonian by Proposition 3.2. The last assertion now follows from the observation that a Lee element for the action on $M$ must be nonzero, because $M$ is strict conformal symplectic.

\section{Convexity properties of the moment map}

The purpose of this section is to prove the convexity theorem, Theorem 1.1. The basic idea is to use Proposition 3.4 to obtain local convexity, and then to apply a local-global principle.

Notation and conventions. Except for Theorem 4.7, in this section $(M, \omega, \theta)$ denotes a connected strict conformal symplectic manifold equipped with a Hamiltonian action of the compact connected Lie group $K$. The (unique) equivariant moment map for the action is denoted by $\Phi: M \rightarrow \mathfrak{k}^{*}$. We assume the action is of Lee type and choose a central Lee element $\zeta$, i.e. an element $\zeta \in \mathfrak{z}(\mathfrak{k})$ such that $\zeta_{M}$ is equal to the Lee vector field $A$. The existence of such an element is guaranteed by Lemma 3.7. We let $\mathcal{H}_{\zeta}$ be the affine hyperplane

$$
\mathcal{H}_{\zeta}=\left\{v \in \mathfrak{k}^{*} \mid\langle v, \zeta\rangle=1\right\}
$$

We fix a maximal torus $T$ of $K$ with Lie algebra $\mathfrak{t}$ and a closed chamber $C$ in the dual space $\mathfrak{t}^{*}$. Since the Lee element $\zeta$ is central, it is contained in the Cartan subalgebra $\mathfrak{t}$. Moreover, $\mathfrak{t}$ has a natural complement in $\mathfrak{k}$ (the sum of the root spaces), and therefore $\mathfrak{t}^{*}$ can be naturally regarded as a subspace of $\mathfrak{k}^{*}$. The inclusion $C \hookrightarrow \mathfrak{k}^{*}$ induces a homeomorphism $C \stackrel{\simeq}{\longrightarrow} \mathfrak{k}^{*} / \operatorname{Ad}^{*}(K)$, which we use to identify $\mathfrak{k}^{*} / \operatorname{Ad}^{*}(K)$ with $C$. We let $q: \mathfrak{k}^{*} \rightarrow C$ be the quotient map and define $\phi=q \circ \Phi: M \rightarrow C$ to be the composition of $\Phi$ with $q$. The moment body $\Delta(M)$ of $M$ is defined by

$$
\Delta(M)=\Phi(M) \cap C=\phi(M) .
$$

Local convexity. Let $\zeta^{\circ} \subset \mathfrak{k}^{*}$ be the annihilator of the line $\mathbb{R} \cdot \zeta$ in $\mathfrak{k}$ spanned by the Lee element. Then $\zeta^{\circ}$ is a hyperplane in $\mathfrak{k}^{*}$, and for any point $m \in M$ we have $\mathcal{H}_{\zeta}=\Phi(m)+\zeta^{\circ}$. Let $\mathcal{H}_{\zeta}^{+}$be the open halfspace

$$
\mathcal{H}_{\zeta}^{+}=\left\{v \in \mathfrak{k}^{*} \mid\langle v, \zeta\rangle>0\right\}
$$

and define the rescaling map $\varrho: \mathcal{H}_{\zeta}^{+} \rightarrow \mathcal{H}_{\zeta}$ by

$$
\varrho(v)=\frac{v}{\langle v, \zeta\rangle}
$$


in other words $\varrho(v)$ is the unique intersection point of the line through $v \in \mathcal{H}_{\zeta}^{+}$with the affine hyperplane $\mathcal{H}_{\zeta}$. The rescaling map extends to a projective linear map

$$
\hat{\varrho}: \mathbb{P}\left(\mathfrak{k}^{*} \oplus \mathbb{R}\right) \longrightarrow \mathbb{P}\left(\mathfrak{k}^{*} \oplus \mathbb{R}\right)
$$

given by $\hat{\varrho}([v, t])=[v,\langle v, \zeta\rangle]$, where $[v, t]$ denotes the line through a nonzero point $(v, t) \in \mathfrak{k}^{*} \oplus \mathbb{R}$. The map $\varrho$ is a projection onto the hyperplane $\{[v, t] \mid\langle v, \zeta\rangle=t\}$, and it is $K$-equivariant, where we let $K$ act by the coadjoint action on $\mathfrak{k}^{*}$ and trivially on $\mathbb{R}$. These facts imply that $\varrho$ has the following properties.

4.2. Lemma. (i) The map @ is open and its fibres are connected.

(ii) The map @ maps convex sets to convex sets and convex polyhedral sets to convex polyhedral sets. Let $\ell=\{t v \mid t>0\}$ be the ray (open halfine) through any $v \in \mathcal{H}_{\zeta}^{+}$. Then $\varrho\left(\ell \cap \mathcal{H}_{\zeta}^{+}\right)$is a ray in $\mathcal{H}_{\zeta}$.

(iii) The affine hyperplane $\mathcal{H}_{\zeta}$ and the halfspace $\mathcal{H}_{\zeta}^{+}$are $\mathrm{Ad}^{*}$-invariant. The map $\varrho$ is $\mathrm{Ad}^{*}$-equivariant and maps $\mathcal{H}_{\zeta}^{+} \cap C$ onto $\mathcal{H}_{\zeta} \cap C$. The diagram

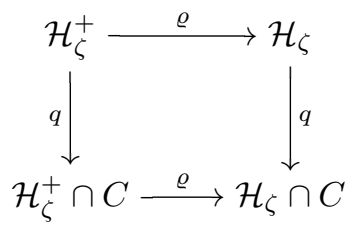

commutes.

We deduce from this the following local convexity theorem.

4.3. Theorem. Assume that $(M, \omega, \theta)$ is strict conformal symplectic and that the $K$-action on $M$ is Hamiltonian and of Lee type. Then for each $m \in M$ there exist a closed convex cone $C_{m}$ in $\mathfrak{t}^{*} \cap \mathcal{H}_{\zeta}$ with apex $\phi(m)$, and a basis of $K$-invariant open neighborhoods $U$ of $m$ such that

(i) the fibres of the map $\left.\phi\right|_{U}$ are connected;

(ii) $\phi: U \rightarrow C_{m}$ is an open map.

Proof. Let $m \in M$ and let $V$ be an equivariant tubular neighborhood of the orbit $K \cdot m$. Choose a function $f \in \mathcal{C}^{\infty}(V)$ as in Proposition 3.4, and let $\Omega=\left.e^{f} \cdot \omega\right|_{V}$ and $\Psi=\left.e^{f} \cdot \Phi\right|_{V}$. Then $(V, \Omega)$ is a symplectic Hamiltonian $K$-manifold with moment map $\Psi$. Let $x \in V$. Since $\Phi(x) \in \mathcal{H}_{\zeta}$ (by Lemma 3.6) and $\Psi(x)$ is a positive scalar multiple of $\Phi(x)$, we have $\Psi(x) \in \mathcal{H}_{\zeta}^{+}$and therefore $\Phi(x)=\varrho(\Psi(x))$. This shows that $\left.\Phi\right|_{V}=\varrho \circ \Psi$. Putting $\psi=q \circ \Psi$ we obtain from Lemma 4.2(iii) that

$$
\left.\phi\right|_{V}=\left.q \circ \Phi\right|_{V}=q \circ \varrho \circ \Psi=\varrho \circ q \circ \Psi=\varrho \circ \psi,
$$

and in particular $\phi(V)=\varrho \circ \psi(V)$ (see Figure 1). The symplectic version of the local convexity theorem [36, Theorem 6.5] asserts that there exist a basis of $K$ invariant open neighborhoods $U \subset V$ of $m$ and a rational convex polyhedral cone $\Delta_{m} \subset \mathfrak{t}^{*} \subset \mathfrak{k}^{*}$ with apex $\psi(m)$ such that $\left.\psi\right|_{U}: U \rightarrow \Delta_{m}$ is an open map with connected fibres. In particular, the set $\psi(U)$ is a neighborhood of $\psi(m)$ in $\Delta_{m}$ and the cone with apex $\psi(m)$ spanned by $\psi(U)$ is equal to $\Delta_{m}$. Define

$$
C_{m}=\varrho\left(\Delta_{m} \cap \mathcal{H}_{\zeta}^{+}\right) \text {. }
$$

Lemma 4.2(ii) shows that $C_{m}$ is a closed convex polyhedral cone, namely the cone with apex $\varrho(\psi(m))=\phi(m)$ spanned by $\varrho(\psi(U))$. Lemma 4.2(i) shows that $\varrho: \Delta_{m} \cap$ 


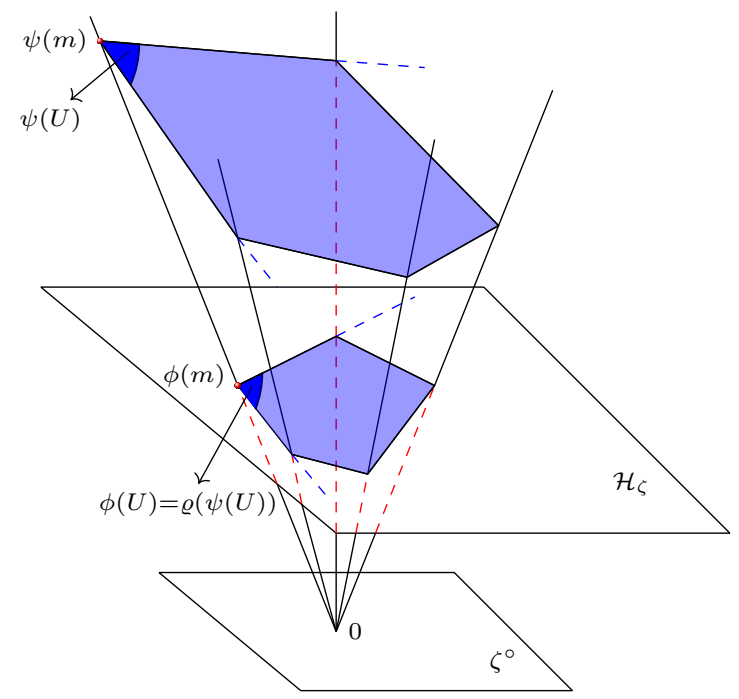

Figure 1. Projecting the local moment cone

$\mathcal{H}_{\zeta}^{+} \rightarrow C_{m}$ is an open map (for the subspace topologies inherited from $\mathcal{H}_{\zeta}^{+}$and $\mathcal{H}_{\zeta}$, respectively). It now follows from (4.4) that $\phi: U \rightarrow C_{m}$ is an open map, which proves (ii).

Let $x \in U$ and put $a=\phi(x)$. Put $A=\varrho^{-1}(a)$; then $A$ is connected by Lemma 4.2(i) and $\left(\left.\phi\right|_{U}\right)^{-1}(a)=\left(\left.\psi\right|_{U}\right)^{-1}(A)$ by (4.4). As the map $\psi: U \rightarrow \Delta_{m} \cap \mathcal{H}_{\zeta}^{+}$ is open, so is the surjective map

$$
\psi: \psi^{-1}(A) \rightarrow A .
$$

Now suppose that $\left(\left.\phi\right|_{U}\right)^{-1}(a)=\left(\left.\psi\right|_{U}\right)^{-1}(A)$ was not connected. Then it is the union of two disjoint nonempty open subsets $W_{1}$ and $W_{2}$. Therefore the sets $\psi\left(W_{1}\right)$ and $\psi\left(W_{2}\right)$ form an open covering of $A$. Thanks to the connectedness of $A$, the intersection $B=\psi\left(W_{1}\right) \cap \psi\left(W_{2}\right)$ is nonempty and therefore $\left(\left.\psi\right|_{U}\right)^{-1}(b)$ is not connected for any $b \in B$. This contradicts the fact that the fibres of the map $\psi: U \rightarrow \Delta_{m}$ are connected, which completes the proof of (i).

From local to global. We will now derive the global convexity theorem by means of a local-global principle. We use the version of the local-global principle due to [17]. Let $X$ be a connected Hausdorff topological space and $E$ a finite dimensional vector space. A continuous map $\Psi: X \rightarrow E$ is said to be locally fiber connected, if every point $x$ in $X$ admits arbitrarily small neighborhoods $U$ such that $\Psi^{-1}(\Psi(u)) \cap$ $U$ is connected for all $u \in U$. We say that a map $x \mapsto C_{x}$ assigning to each point $x \in X$ a closed convex cone $C_{x} \subset E$ with apex $\Psi(x)$ is a system of local convexity data if the following conditions hold:

(i) for each $x$ there exists an arbitrarily small open neighborhood $U_{x}$ such that the map $\Psi: U_{x} \rightarrow C_{x}$ is open;

(ii) $\Psi^{-1}(\Psi(u)) \cap U_{x}$ is connected for all $u \in U_{x}$.

4.5. Theorem ([17, Theorem 3.10]). Suppose that $\Psi: X \rightarrow E$ is a proper, locally fiber connected map with the local convexity data $\left(C_{x}\right)_{x \in X}$. Then the fibres of $\Psi$ are 
connected and $\Psi: X \rightarrow \Psi(X)$ is an open map; moreover, $\Psi(X)$ is a closed convex polyhedral subset of $E$.

Proof of Theorem 1.1. Theorem 4.3 shows that the family of cones $\left(C_{m}\right)_{m \in M}$ is a system of local convexity data for the map $\phi: M \rightarrow C$. On account of the properness of the moment map $\Phi$ and the quotient map $q$, the map $\phi=q \circ \Phi: M \rightarrow C$ is proper. Theorem 1.1 now follows immediately from Theorem 4.5.

4.6. Remarks. (i) Recall that a closed convex subset of a finite dimensional vector space can be expressed as the intersection of all its supporting halfplanes. As shown in $[17, \S 1])$, we have

$$
C_{m}=\phi(m)+L_{\phi(m)}(\phi(M)),
$$

where $L_{\phi(m)}(\phi(M))$ is the intersection of all supporting halfplanes of $\phi(M)$ at the point $\phi(m)$. It follows that

$$
\Delta(M)=\bigcap_{m \in M} C_{m}
$$

which is an intersection of a locally finite family of convex cones.

(ii) Let $a \in \mathcal{C}^{\infty}\left(M, \mathbb{R}_{>0}\right)$. A gauge transformation $a \cdot(\omega, \theta)$ changes the moment map to $a \Phi$, which usually destroys the convexity of the image. Thus the moment body $\Delta(M)$ is not a conformal invariant of $M$.

(iii) The above proof does not tell us whether the moment body $\Delta(M)$ is semirational or not. The reason lies in the fact the the rescaling map does not preserve the rationality of convex cones in general. However, for strict conformal symplectic manifolds of the first kind we will state a necessary and sufficient condition for the moment body to be semirational; see Theorem 6.5.

We finish this section with a counterpart of Theorem 1.1 for global conformal symplectic manifolds.

4.7. Theorem. Let $(M, \omega, \theta=d f)$ be a connected global conformal symplectic manifold equipped with a $K$-action which leaves the forms $\omega$ and $\theta$ invariant. Suppose that the action is Hamiltonian and that the moment map $\Phi$ for the action is proper. Suppose also that the image of $\Phi$ is contained in the hyperplane $\mathcal{H}_{\zeta}$ for some $\zeta \in \mathfrak{k}$. Then the conclusions of the convexity theorem, Theorem 1.1, hold for M.

Proof. As in the proof of Lemma 3.6 one sees that the action is of Lee type. As in the proof of Lemma 3.7 one shows that the Lee element $\zeta$ can be assumed to be central. The remainder of the proof is identical to that of Theorem 1.1.

The assumptions of this theorem apply for instance if $M$ is a closed $K$-invariant global conformal symplectic submanifold of a strict conformal symplectic manifold furnished with a Hamiltonian $K$-action.

\section{SympleCtic, CONFORMAl SyMPleCtiC, AND PRESYMPLECTIC CONVEXITY}

In this section we explain how the conformal symplectic convexity theorem of the previous section relates to Kirwan's symplectic convexity theorem [20] as well as to the presymplectic convexity theorem of [30].

The relationship to symplectic convexity arises from the fact that a conformal symplectic manifold $M$ has symplectic covering manifolds $\tilde{M}$, and that a Hamiltonian $K$-action on $M$ lifts to a Hamiltonian action of a covering group $\tilde{K}$ on $\tilde{M}$. 
Theorems 5.2 and 5.4 below assert that in good cases the moment body $\Delta(\tilde{M})$ is a cone over the moment body $\Delta(M)$, and that $\Delta(M)$ is the intersection of $\Delta(\tilde{M})$ with an affine hyperplane. These facts generalize results for torus actions obtained in [5].

The relationship to presymplectic convexity comes from the fact that the Lee form of a conformal symplectic manifold $M$ defines a singular foliation, which we will call the Lee foliation, whose regular leaves are of codimension 1 and carry natural presymplectic structures. The $K$-action preserves each leaf $L$, provided that the action is of Lee type. We will see that the Lee type condition is equivalent to a leafwise transitivity condition for the action on $L$. Theorem 5.7 below asserts a form of Reeb stability for the Lee foliation and also that the moment body $\Delta(L)$ is equal to $\Delta(M)$ for all leaves $L$. In particular all leaves have the same moment body. This fact was observed for Vaisman manifolds in [5].

Notation and conventions. In this section $(M, \omega, \theta)$ denotes a connected strict conformal symplectic manifold equipped with a Hamiltonian action of the compact connected Lie group $K$, which has equivariant moment map $\Phi: M \rightarrow \mathfrak{k}^{*}$. The moment body of $M$ is denoted by $\Delta(M)=\Phi(M) \cap C$, where $C$ is a fixed closed Weyl chamber in $\mathfrak{t}^{*}$, the dual of the Lie algebra $\mathfrak{t}$ of a maximal torus $T$ of $K$.

Definitions. A presymplectic form on a manifold $P$ is a closed 2-form $\sigma \in \Omega^{2}(P)$ of constant rank. The kernel of a presymplectic form $\sigma$ on $P$ is an involutive subbundle of $T P$, which generates a (regular) foliation $\mathcal{F}_{\sigma}$ called the null foliation of $\sigma$.

Let $\mathcal{F}$ be a foliation of a manifold $P$. Let $\mathfrak{X}(\mathcal{F})$ be the Lie algebra of vector fields tangent to $\mathcal{F}$. A vector field $X \in \mathfrak{X}(M)$ is foliate if $[X, Y] \in \mathfrak{X}(\mathcal{F})$ for all $Y \in \mathfrak{X}(\mathcal{F})$. A smooth map $\phi: X \rightarrow X$ is foliate if $\phi(L)$ is contained in a leaf of $\mathcal{F}$ for every leaf $L$ of $\mathcal{F}$. A foliate vector field generates a flow consisting of (local) foliate diffeomorphisms. Suppose that a Lie group $G$ acts smoothly on $P$ by foliate transformations. Let $\mathfrak{n}_{\mathcal{F}}$ be the set of all $\xi \in \mathfrak{g}=\operatorname{Lie}(G)$ such that the induced vector field $\xi_{P}$ is tangent to $\mathcal{F}$. Then $\mathfrak{n}_{\mathcal{F}}$ is an ideal of $\mathfrak{g}$ called the null ideal of the action. (See $[30, \S 2.5]$.) The null subgroup is the immersed (not necessarily closed) normal subgroup $N_{\mathcal{F}}$ of $G$ generated by $\exp \left(\mathfrak{n}_{\mathcal{F}}\right)$. The $G$-action on $(P, \mathcal{F})$ is clean if

$$
T_{p}(G \cdot p) \cap T_{p} \mathcal{F}=T_{p}\left(N_{\mathcal{F}} \cdot p\right)
$$

for every $p \in P$. The $G$-action is leafwise transitive if $T_{p} \mathcal{F}=T_{p}\left(N_{\mathcal{F}} \cdot p\right)$ for every $p \in P$. Clearly, if the action is leafwise transitive, it is clean.

Presentations. See $[5, \S 2.1]$ for the following definitions and facts. A presentation of the strict conformal symplectic manifold $(M, \omega, \theta)$ is a connected symplectic manifold $(\tilde{M}, \tilde{\omega})$ equipped with a Galois covering map

$$
p: \tilde{M} \longrightarrow M
$$

with covering group $\Gamma$, a group homomorphism $\chi: \Gamma \rightarrow(\mathbb{R},+)$, and a function $\tilde{f} \in \mathcal{C}^{\infty}(\tilde{M})$ called the potential of the presentation, which is required to have the following properties: 0 is a regular value of $\tilde{f}$;

$$
\tilde{\omega}=e^{\tilde{f}} p^{*} \omega ; \quad d \tilde{f}=p^{*} \theta ; \quad \text { and } \quad \gamma^{*} \tilde{f}=\tilde{f}+\chi(\gamma) \quad \text { for all } \gamma \in \Gamma .
$$

Given a presentation $\tilde{M}$, the potential $\tilde{f}$ is unique up to an additive constant $c$ and the symplectic form $\tilde{\omega}$ is unique up to a multiplicative constant $e^{c}$. The 
condition that 0 should be a regular value of $\tilde{f}$ is not imposed in [5], but is easy to fulfil: pick a regular value $-c$ of $\tilde{f}$ and replace $\tilde{f}$ with $\tilde{f}+c$ and $\tilde{\omega}$ with $e^{c} \tilde{\omega}$ to make 0 a regular value. Presentations of $M$ exist, e.g. the universal cover of $M$. A presentation $\widehat{M}$ of $M$ is minimal if for every other presentation $\tilde{M}$ there is a morphism of covering spaces $\tilde{M} \rightarrow \widehat{M}$. Minimal presentations exist and are unique up to covering space isomorphisms. A presentation $\widehat{M}$ is minimal if and only if the corresponding homomorphism $\chi: \Gamma \rightarrow \mathbb{R}$ is injective.

Conversely, suppose we are given a connected symplectic manifold $(\tilde{M}, \tilde{\omega})$, a discrete group $\Gamma$ acting properly and freely on $\tilde{M}$, a homomorphism $\chi: \Gamma \rightarrow \mathbb{R}$, and a function $\tilde{f}$ such that $\gamma^{*} \tilde{\omega}=e^{\chi(\gamma)} \tilde{\omega}$ and $\gamma^{*} \tilde{f}=\tilde{f}+\chi(\gamma)$ for all $\gamma \in \Gamma$. Then the forms $e^{-\tilde{f}} \tilde{\omega}$ and $d \tilde{f}$ are $\Gamma$-invariant, and therefore descend to forms $\omega$ and $\theta$ on $M=\tilde{M} / \Gamma$. The pair $(\omega, \theta)$ is a conformal symplectic structure on $M$ and $\tilde{M}$ is a presentation of $M$.

The period homomorphism $\operatorname{per}_{\theta}: \pi_{1}(M) \rightarrow \mathbb{R}$ is defined by $\operatorname{per}_{\theta}([c])=\int_{c} \theta$; it depends only on the Lee class $[\theta] \in H^{1}(M ; \mathbb{R})$. The period group of $\theta$ is the subgroup $\operatorname{Per}_{\theta}=\operatorname{per}_{\theta}\left(\pi_{1}(M)\right) \cong \mathbb{Z}^{k}$ of $\mathbb{R}$; the rank of $\theta$, or of $M$, is the rank $k$ of the period group. We have $\operatorname{Per}_{\theta}=\chi(\Gamma)$ for any presentation of $M$ and $1 \leq \operatorname{rank}(M) \leq b_{1}(M)$.

The moment cone of a presentation. Let $(\tilde{M}, \tilde{\omega}, \Gamma, \chi, \tilde{f})$ be a presentation of $(M, \omega, \theta)$. The $K$-action on $M$ lifts to an action of an appropriate covering group $\tilde{K}$ on $\tilde{M}$, which commutes with the $\Gamma$-action. By [5, Remark 4.4] the $\tilde{K}$-action on the symplectic manifold $(\tilde{M}, \tilde{\omega})$ is Hamiltonian with equivariant moment map given by

$$
\tilde{\Phi}=e^{\tilde{f}} p^{*} \Phi .
$$

By [5, Lemma 4.2], if $\tilde{M}$ is minimal we can take $\tilde{K}=K$. We denote by $\Delta(\tilde{M})=$ $\tilde{\Phi}(\tilde{M}) \cap C$ the moment body of $\tilde{M}$ with respect to the Hamiltonian $\tilde{K}$-action. Applying a conformal transformation $a \cdot(\omega, \theta)$ to the conformal symplectic structure, where $a$ is a positive function on $M$, has the effect of replacing the potential $\tilde{f}$ by $\tilde{f}-\log p^{*} a$, but changes neither the symplectic form $\tilde{\omega}$ nor the moment map $\tilde{\Phi}$, and therefore has no effect on the moment body $\Delta(\tilde{M})$. On the other hand, shifting the potential by a constant $c$ has the effect of dilating the moment body $\Delta(\tilde{M})$ by the positive constant $e^{c}$. Up to such dilations, the moment body is independent of the presentation $\tilde{M}$. Thus the moment body $\Delta(\tilde{M})$, unlike the moment body $\Delta(M)$ of $M$ itself, is a conformal invariant of $M$, up to a positive multiplicative constant $e^{c}$. In fact, the equivariance property

$$
\gamma^{*} \tilde{\Phi}=e^{\chi(\gamma)} \tilde{\Phi}
$$

for all $\gamma \in \Gamma$ shows that $\Delta(\tilde{M})$ is preserved by all dilations in the subgroup $\exp (\chi(\Gamma))$ of $\mathbb{R}_{>0}$. Under what conditions is $\Delta(\tilde{M})$ preserved by all dilations in $\mathbb{R}_{>0}$ ? There is a natural dichotomy according to whether $M$ has rank 1 (when $\operatorname{Per}_{\theta} \cong \mathbb{Z}$ is discrete in $\mathbb{R}$ ) or rank $\geq 2$ (when $\operatorname{Per}_{\theta}$ is dense in $\left.\mathbb{R}\right)$. The following two results extend to the nonabelian case Theorems 5.13 and 5.15 of [5].

5.2. Theorem. Let $(M, \omega, \theta)$ be a compact connected strict conformal symplectic manifold of rank 1 equipped with a Hamiltonian $K$-action. Let $(\tilde{M}, \tilde{\omega}, \Gamma, \chi, \tilde{f})$ be a presentation of $(M, \omega, \theta)$.

(i) Suppose that $0 \notin \Delta(M)$, or equivalently $0 \notin \Delta(\tilde{M})$. Then $\Delta(\tilde{M}) \cup\{0\}$ is a rational convex polyhedral cone and $\Delta(\tilde{M})=\mathbb{R}_{>0} \cdot \Delta(M)$. 
(ii) Suppose that the $K$-action on $(M, \omega, \theta)$ is of Lee type with central Lee element $\zeta$. Then $\Delta(M)=\Delta(\tilde{M}) \cap \mathcal{H}_{\zeta}$. Hence the convex polytope $\Delta(M)$ is semirational if and only if the line $\mathbb{R} \zeta$ in $\mathfrak{t}^{*}$ is rational.

Proof. (i) The moment body $\Delta(\tilde{M})$ being independent of the presentation up to a dilation, we may, and will, assume without loss of generality that the presentation $\tilde{M}$ is minimal. The assumptions $0 \notin \Phi(M), M$ has $\operatorname{rank} 1$, and $\tilde{M}$ is minimal imply that the moment map $\tilde{\Phi}$ is proper when viewed as a map $\tilde{M} \rightarrow \mathfrak{k}^{*} \backslash\{0\}$. (See [5, Lemma 5.14]. This lemma asserts that $\tilde{\Phi}$ is proper viewed as a map $\tilde{M} \rightarrow \mathfrak{k}^{*}$, but that is incorrect.) Therefore, by the version of the Kirwan convexity theorem due to [26], the moment body $\Delta(\tilde{M})$ is a closed convex polyhedral subset of the punctured chamber $C \backslash\{0\}$. It now follows from the scaling property (5.1) that $\Delta(\tilde{M})$ is $\mathbb{R}_{>0}$-invariant. Hence its closure $\Delta(\tilde{M}) \cup\{0\}$ is a closed convex polyhedral cone contained in $C$. The facets of $\Delta(\tilde{M})$ have rational normal vectors, so the cone $\Delta(\tilde{M}) \cup\{0\}$ is rational.

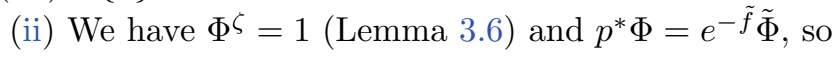

$$
\Delta(M)=\varrho(\Delta(\tilde{M})),
$$

where $\varrho$ is the projection (4.1). On the other hand, $\Phi^{\zeta}=1$ implies $0 \notin \Phi(M)$, so $\Delta(\tilde{M})=\mathbb{R}_{>0} \cdot \Delta(M)$ by (i), and hence $\Delta(M)=\Delta(\tilde{M}) \cap \mathcal{H}_{\zeta}$. The last assertion now follows from (i).

If $M$ has rank $\geq 2$ the moment map $\tilde{\Phi}$ is not proper, but nevertheless we get a slightly weaker conclusion under a stronger hypothesis.

5.4. Theorem. Let $(M, \omega, \theta)$ be a compact connected strict conformal symplectic manifold of rank $\geq 2$ equipped with a Hamiltonian $K$-action. Let $(\tilde{M}, \tilde{\omega}, \Gamma, \chi, \tilde{f})$ be a presentation of $(M, \omega, \theta)$.

(i) Suppose that $0 \notin \Delta(M)$. Suppose also that the Lee form $\theta$ vanishes nowhere, or equivalently that the potential $\tilde{f}$ has no singular values. Then $\Delta(\tilde{M})=\mathbb{R}_{>0} \cdot \Delta(M)$.

(ii) Suppose that the $K$-action on $(M, \omega, \theta)$ is of Lee type with central Lee element $\zeta$. Suppose also that for some $a \in \mathcal{C}^{\infty}\left(M, \mathbb{R}_{>0}\right)$ the gauge-transformed Lee form $\theta-d \log$ a vanishes nowhere. Then $\Delta(\tilde{M}) \cup\{0\}$ is a convex polyhedral cone and $\Delta(M)=\Delta(\tilde{M}) \cap \mathcal{H}_{\zeta}$.

Proof. (i) The following argument adapts the proof of [5, Theorem 5.15]. Let $\tilde{x}_{0} \in \tilde{M}$. Because the rank of $M$ is $\geq 2$, the ray $\mathbb{R}_{>0} \cdot \tilde{\Phi}\left(\tilde{x}_{0}\right)$ intersects the image $\tilde{\Phi}(\tilde{M})$ in a dense subset of the ray. We will argue that the intersection is an open subset of the ray. Let $x_{0}=p\left(\tilde{x}_{0}\right) \in M$. Let $H$ be the identity component of the stabilizer $K_{x_{0}}$ and let $\mathfrak{h}=\operatorname{Lie}(H)=\operatorname{Lie}\left(K_{x_{0}}\right)=\operatorname{Lie}\left(\tilde{K}_{\tilde{x}_{0}}\right)$. Define

$$
\begin{array}{rlrl}
M^{\mathfrak{h}} & =\left\{x \in M \mid \operatorname{Lie}\left(K_{x}\right) \supseteq \mathfrak{h}\right\}, & & M_{\mathfrak{h}}=\left\{x \in M \mid \operatorname{Lie}\left(K_{x}\right)=\mathfrak{h}\right\}, \\
\tilde{M}^{\mathfrak{h}}=\left\{\tilde{x} \in \tilde{M} \mid \operatorname{Lie}\left(\tilde{K}_{\tilde{x}}\right) \supseteq \mathfrak{h}\right\}, & \tilde{M}_{\mathfrak{h}}=\left\{\tilde{x} \in \tilde{M} \mid \operatorname{Lie}\left(\tilde{K}_{\tilde{x}}\right)=\mathfrak{h}\right\} .
\end{array}
$$

It is an elementary fact that $M^{\mathfrak{h}}$, resp. $\tilde{M}^{\mathfrak{h}}$, is a closed submanifold of $M$, resp. $\tilde{M}$ (which may have connected components of varying dimensions), and that $M_{\mathfrak{h}}$, resp. $\tilde{M}_{\mathfrak{h}}$, is an open submanifold of $M^{\mathfrak{h}}$, resp. $\tilde{M}^{\mathfrak{h}}$. Moreover, the submanifold $\tilde{M}^{\mathfrak{h}}$ is symplectic. It follows that $M^{\mathfrak{h}}=p\left(\tilde{M}^{\mathfrak{h}}\right)$ is a closed conformal symplectic submanifold of $M$ with conformal symplectic structure $\left(\omega^{\mathfrak{h}}=\left.\omega\right|_{M^{\mathfrak{h}}}, \theta^{\mathfrak{h}}=\left.\theta\right|_{M^{\mathfrak{h}}}\right)$. If 
$V$ denotes the tangent space $T_{x} M$ at some point $x \in M^{\mathfrak{h}}$, then the tangent space to $M^{\mathfrak{h}}$ is $T_{x} M^{\mathfrak{h}}=V^{\mathfrak{h}}$, the subspace of $\mathfrak{h}$-fixed vectors. This subspace has a natural complement: we have $V=V^{\mathfrak{h}} \oplus \mathfrak{h} V$, where $\mathfrak{h} V$ is defined as the subspace spanned by all vectors $\eta \cdot v=\left[\eta_{M}, v\right]$ with $\eta \in \mathfrak{h}$ and $v \in V$. Thus the normal bundle $N=N_{M} M^{\mathfrak{h}}$ of $M^{\mathfrak{h}}$ is naturally a subbundle of $\left.T M\right|_{M^{\mathfrak{h}}}$. For $v \in V$ and $\eta \in \mathfrak{h}$ we have

$$
\theta_{x}(\eta \cdot v)=\omega\left(A_{x}, \eta \cdot v\right)=-\omega\left(\eta \cdot A_{x}, v\right)=0,
$$

because $\omega$ and the Lee vector field $A$ are $K$-invariant. Therefore $\left.\theta\right|_{N}=0$. As in [5, Corollary 5.10] this implies that the Lee form $\theta^{\mathfrak{h}}$ is not exact. For if $\theta^{\mathfrak{h}}=d f$ for some $f \in \mathcal{C}^{\infty}\left(M^{\mathfrak{h}}\right)$, then $\theta_{x}^{\mathfrak{h}}=0$ for any $x$ in the compact manifold $M^{\mathfrak{h}}$ where $f$ attains an extremum. Hence we would have $\theta_{x}=0$, which contradicts the hypothesis on $\theta$. This shows that the conformal symplectic manifold $M^{\mathfrak{h}}$ is strict. Since $\eta_{M}=0$ on $M^{\mathfrak{h}}$ for all $\eta \in \mathfrak{h}$, it follows from Proposition 2.4 that $\Phi^{\eta}=0$ on $M^{\mathfrak{h}}$ for all $\eta \in \mathfrak{h}$; in other words $\Phi\left(M^{\mathfrak{h}}\right) \subseteq \mathfrak{h}^{\circ}$. For $h \in H$ and $x \in M^{\mathfrak{h}}$ we have $\Phi(x)=\Phi(h x)=\operatorname{Ad}_{h}^{*} \Phi(x)$, and therefore

$$
\Phi\left(M^{\mathfrak{h}}\right) \subseteq \mathfrak{h}^{\circ} \cap \mathfrak{k}^{H} .
$$

Let $N_{K}(H)$ be the normalizer of $H, L=N_{K}(H) / H$, and $\mathfrak{l}=\operatorname{Lie}(L)$. The subspace $\mathfrak{h}^{\circ} \cap \mathfrak{k}^{H}$ of $\mathfrak{k}^{*}$ is naturally isomorphic to $\mathfrak{l}^{*}$, and $\Phi$ restricts to a moment map for the $L$-action on $M^{\mathfrak{h}}$; cf. [37, §3]. Likewise, $\tilde{\Phi}$ maps $\tilde{M}^{\mathfrak{h}}$ to $\mathfrak{l}^{*}$ and the restriction of $\tilde{\Phi}$ to $\tilde{M}^{\mathfrak{h}}$,

$$
\tilde{\Phi}^{\mathfrak{h}}: \tilde{M}^{\mathfrak{h}} \longrightarrow \mathfrak{l}^{*},
$$

is a moment map for the $L$-action on $\tilde{M}^{\mathfrak{h}}$. The $L$-action on the open submanifold $\tilde{M}_{\mathfrak{h}}$ of $\tilde{M}^{\mathfrak{h}}$ is locally free, so $\tilde{\Phi}^{\mathfrak{h}}$ restricted to $\tilde{M}_{\mathfrak{h}}$ is a submersion. Since $\tilde{x}_{0} \in \tilde{M}_{\mathfrak{h}}$, it follows that the image $\tilde{\Phi}\left(\tilde{M}_{\mathfrak{h}}\right)$ intersects the ray through $\tilde{\Phi}\left(\tilde{x}_{0}\right)$ in an open subset.

(ii) Let $\Delta_{a}(M)$ be the moment body of $M$ with respect to the moment map $a \Phi$. It follows from (i) that

$$
\Delta(\tilde{M})=\mathbb{R}_{>0} \cdot \Delta_{a}(M)=\mathbb{R}_{>0} \cdot \Delta(\tilde{M}) .
$$

Therefore, by (5.3), $\Delta(M)=\varrho(\Delta(\tilde{M}))=\Delta(\tilde{M}) \cap \mathcal{H}_{\zeta}$. Hence also $\Delta(\tilde{M})=\mathbb{R}_{>0}$. $\Delta(M)$. By Theorem 1.1, $\Delta(M)$ is a convex polytope, so $\Delta(\tilde{M}) \cup\{0\}$ is a convex polyhedral cone.

The Lee foliation. For $x$ and $y \in M$ define $x \sim y$ if there exists a smooth path $\gamma$ joining $x$ to $y$ and satisfying $\theta\left(\gamma^{\prime}(t)\right)=0$ for all $t$. The relation $\sim$ is an equivalence relation on $M$, whose equivalence classes we call the leaves of the Lee foliation $\mathcal{F}_{\theta}$. The leaves may be singular at points where $\theta$ vanishes, but on the open subset

$$
U_{\theta}=\left\{x \in M \mid \theta_{x} \neq 0\right\}
$$

the foliation $\mathcal{F}_{\theta}$ is a regular foliation, whose tangent bundle is $T \mathcal{F}_{\theta}=\operatorname{ker}(\theta)$ and whose leaves are immersed submanifolds of codimension 1 . For every leaf $L$ the form $\theta$ vanishes on $L \cap U_{\theta}$, so the 2 -form $\omega_{L}=\left.\omega\right|_{L \cap U_{\theta}}$ is closed. The Lee vector field $A$ satisfies $\iota(A) \theta=0$, and therefore restricts to a vector field $A_{L}$ on $L \cap U_{\theta}$, which spans the null foliation of $\omega_{L}$. Thus we see that the regular part of each leaf is a presymplectic manifold of corank 1 .

We assert that a Hamiltonian $K$-action preserves each leaf of the Lee foliation and that, if the action is of Lee type, the action on each leaf is leafwise transitive. 
5.5. Lemma. Let $(M, \omega, \theta)$ be a connected strict conformal symplectic manifold equipped with a Hamiltonian $K$-action. Then $K \cdot L=L$ for every leaf $L$ of $\mathcal{F}_{\theta}$, and the $K$-action on the presymplectic manifold $L \cap U_{\theta}$ is Hamiltonian with moment map $\Phi_{L}=\left.\Phi\right|_{L \cap U_{\theta}}$. If the action is of Lee type, then the action on $L \cap U_{\theta}$ is leafwise transitive relative to the null foliation of $\omega_{L}$.

Proof. Let $\xi \in \mathfrak{k}$. From the fact that $\theta$ is basic (Proposition 3.2) it follows that $\theta\left(\xi_{M}\right)=0$. Therefore $T_{x}(K \cdot x)$ is tangent to $L$ for every $x \in L$, and hence $K \cdot x \subseteq L$. On $M$ we have $d_{\theta} \Phi^{\xi}=\iota\left(\xi_{M}\right) \omega$, so on $L \cap U_{\theta}$ we have $d \Phi_{L}^{\xi}=\iota\left(\xi_{L}\right) \omega_{L}$ for all $\xi \in \mathfrak{k}$. Thus $\Phi_{L}$ is a moment map for the $K$-action on $L \cap U_{\theta}$. If $\zeta$ is a Lee element for the action, the null foliation of $\omega_{L}$ is spanned by $A_{L}=\zeta_{L}$, so the $K$-action on $L \cap U_{\theta}$ is leafwise transitive.

If $\theta$ vanishes nowhere, all the leaves of $\mathcal{F}_{\theta}$ are nonsingular, and we can choose a vector field $B \in \mathfrak{X}(M)$ satisfying $\theta(B)=1$. Such a vector field is unique up to a vector field tangent to the Lee foliation and its orbits are transversals to the Lee foliation. We claim that $\theta$ is $B$-invariant and that $B$ is foliate.

5.6. Lemma. Let $(M, \omega, \theta)$ be a connected strict conformal symplectic manifold. Suppose the Lee form $\theta$ vanishes nowhere and choose a vector field $B$ satisfying $\iota(B) \theta=1$. Then $L(B) \theta=0$ and $B$ is $\mathcal{F}_{\theta}$-foliate. Let $\tilde{B}$ be the lift of $B$ to $a$ presentation $(\tilde{M}, \tilde{\omega}, \Gamma, \chi, \tilde{f})$. Then $L(\tilde{B}) \tilde{f}=1$.

Proof. It follows from $\iota(B) \theta=1$ that $L(B) \theta=[\iota(B), d] \theta=d \iota(B) \theta=0$. Let $X \in \mathfrak{X}\left(\mathcal{F}_{\theta}\right)$, i.e. $\iota(X) \theta=0$. Then

$$
\iota([B, X]) \theta=L(B) \iota(X) \theta-\iota(X) L(B) \theta=0,
$$

so $[B, X] \in \mathfrak{X}\left(\mathcal{F}_{\theta}\right)$. Hence $B$ is foliate. It follows from $\iota(B) \theta=1$ that $\iota(\tilde{B}) d \tilde{f}=1$ and hence that $L(\tilde{B}) \tilde{f}=1$.

The vector field $B$ in this lemma can be averaged over $K$ to be made $K$-invariant. If $B$ is complete, its flow maps leaves of $\mathcal{F}_{\theta}$ to leaves of $\mathcal{F}_{\theta}$ in a $K$-equivariant fashion. Lifting the flow of $B$ to the minimal presentation $\tilde{M}$ of $M$ induces a diffeomorphism $\tilde{M} \cong \mathbb{R} \times L$, where $L$ is any leaf of $\mathcal{F}_{\theta}$, and gives the following version of the Reeb stability theorem (see e.g. [31, Ch. 2]) for the Lee foliation.

5.7. Theorem. Let $(M, \omega, \theta)$ be a connected strict conformal symplectic manifold equipped with a Hamiltonian $K$-action with moment map $\Phi$. Let $(\tilde{M}, \tilde{\omega}, \Gamma, \chi, \tilde{f})$ be the minimal presentation of $(M, \omega, \theta)$. Suppose also that the Lee form $\theta$ vanishes nowhere and that there exists a complete $K$-invariant vector field $B$ satisfying $\iota(B) \theta=1$.

(i) Let $\tilde{\mathcal{F}}_{\theta}$ be the lift of the Lee foliation $\mathcal{F}_{\theta}$ to $\tilde{M}$. The leaves of $\tilde{\mathcal{F}}_{\theta}$ are the level sets of the potential $\tilde{f}: \tilde{M} \rightarrow \mathbb{R}$. For each leaf $\tilde{L}$ of $\tilde{\mathcal{F}}_{\theta}$ the covering map p: $\tilde{M} \rightarrow M$ restricts to a diffeomorphism $\tilde{L} \rightarrow L$, where $L$ is a leaf of $\mathcal{F}_{\theta}$.

(ii) Let $\tilde{B}$ be the lift of $B$ to $\tilde{M}$ and let $F: \mathbb{R} \times \tilde{M} \rightarrow \tilde{M}$ be the flow of $\tilde{B}$. Let $\tilde{L}_{0}=\tilde{f}^{-1}(0), L_{0}=p\left(\tilde{L}_{0}\right)$, and $q: L_{0} \rightarrow \tilde{L}_{0}$ the inverse of $p: \tilde{L}_{0} \rightarrow L_{0}$. Define $F_{0}: \mathbb{R} \times L_{0} \rightarrow \tilde{M}$ by $F_{0}(t, x)=F(0, q(x))$. Equip $\mathbb{R} \times L_{0}$ with the foliation given by the fibres of the projection $\mathrm{pr}_{1}: \mathbb{R} \times L_{0} \rightarrow \mathbb{R}$. Then $F_{0}$ is a K-equivariant foliate diffeomorphism. The potential $\tilde{f}: \tilde{M} \rightarrow \mathbb{R}$ descends to a continuous map $f: M \cong \tilde{M} / \Gamma \rightarrow \mathbb{R} / \Gamma$, whose fibres are the 
leaves of $\mathcal{F}_{\theta}$. In other words, we have the following commutative diagram, in which the top row consists of foliated manifolds and foliate maps and the bottom row consists of the respective leaf spaces:

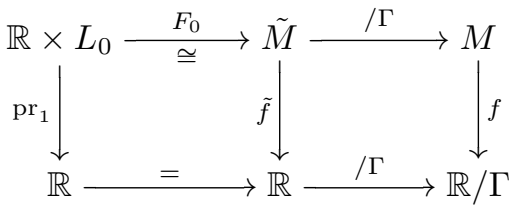

The formula $\gamma * x=p(F(-\chi(\gamma), \gamma \cdot q(x)))$ for $\gamma \in \Gamma$ and $x \in L_{0}$ defines an action of $\Gamma$ on the leaf $L_{0}$. The map $F_{0}$ is equivariant with respect to the diagonal $\Gamma$-action, so $M$ is diffeomorphic to the quotient $\left(\mathbb{R} \times L_{0}\right) / \Gamma$.

(iii) If $\operatorname{rank}(M)=1$ every leaf of $\mathcal{F}_{\theta}$ is closed. If $\operatorname{rank}(M) \geq 2$ every leaf of $\mathcal{F}_{\theta}$ is dense.

(iv) Suppose the $K$-action on $M$ is of Lee type with central Lee element $\zeta \in \mathfrak{z}(\mathfrak{k})$. Let $L$ be any leaf of $\mathcal{F}_{\theta}$ and let $\Delta(L)=\Phi(L) \cap C$ be the moment body of the presymplectic Hamiltonian $K$-manifold $L$. Then $\Delta(L)=\Delta(M)$. In particular $\Delta(L)$ is independent of the leaf $L$ and, if $\Phi$ is proper, is a convex polyhedral set.

Proof. (i)-(ii) Since $p^{*} \theta=d \tilde{f}$, the leaves of $\tilde{\mathcal{F}}_{\theta}$ are the connected components of the level surfaces of $\tilde{f}$. The formula $\gamma^{*} f=f+\chi(\gamma)$ and the injectivity of $\chi: \Gamma \rightarrow \mathbb{R}$ show that the restriction of $p$ to each level surface of $\tilde{f}$ is injective. Thus $p$ maps each leaf of $\tilde{\mathcal{F}}_{\theta}$ diffeomorphically to a leaf of $\mathcal{F}_{\theta}$. We must show that the level sets of $\tilde{f}$ are connected. Since $B$ is complete, so is $\tilde{B}$. It follows from $L(\tilde{B}) \tilde{f}=1$ (Lemma 5.6) that

$$
\frac{d}{d t} \tilde{f}(F(t, \tilde{x}))=1, \quad \tilde{f}(F(0, \tilde{x}))=\tilde{f}(\tilde{x})
$$

for all $\tilde{x} \in \tilde{M}$, whence $\tilde{f}(F(t, \tilde{x}))=t+\tilde{f}(\tilde{x})$ for all $t \in \mathbb{R}$. This shows that $F_{0}$ is a diffeomorphism and that the left square in the diagram (5.8) commutes. Since $\tilde{B}$ is $\tilde{\mathcal{F}}_{\theta}$-foliate and $K$-invariant, $F_{0}$ is $\tilde{\mathcal{F}}_{\theta}$-foliate and $K$-equivariant. By assumption $\tilde{M}$ is connected, and therefore so is $\tilde{L}_{0}$ and every other level set of $\tilde{f}$. The potential $\tilde{f}$ is $\Gamma$-equivariant and therefore descends to a continuous $f: M \rightarrow \mathbb{R} / \Gamma$, which makes the square on the right in (5.8) commute. The flow $F$ is $\Gamma$-equivariant, so it follows from the flow law that

$$
F(\chi(\gamma), \gamma * \tilde{x})=F(\chi(\gamma), F(-\chi(\gamma), \gamma \cdot \tilde{x}))=\gamma \cdot F(\chi(\gamma), F(-\chi(\gamma), \tilde{x}))=\gamma \cdot F(0, \tilde{x})
$$

for all $\gamma \in \Gamma$ and $\tilde{x} \in \tilde{L}_{0}$, which proves the last two assertions of (ii).

(iii) The leaves of $\mathcal{F}_{\theta}$ are the fibres of $f: M \rightarrow \mathbb{R} / \Gamma$. If $\operatorname{rank}(M)=1$ we have $\mathbb{R} / \Gamma \cong \mathbb{S}^{1}$ and $f$ is a smooth locally trivial fibre bundle, so the fibres are closed. Now let $\operatorname{rank}(M) \geq 2$. For $t \in \mathbb{R}$ let $\tilde{L}_{t}$ be the leaf $\tilde{f}^{-1}(t)$ of $\tilde{\mathcal{F}}_{\theta}$ and let $L_{t}$ be the leaf $p\left(\tilde{L}_{t}\right)$ of $\mathcal{F}_{\theta}$. The inverse image of $L_{t}$ in $\mathbb{R} \times L_{0}$ is

$$
\left(p \circ F_{0}\right)^{-1}\left(L_{t}\right)=\Gamma \cdot\left(\{t\} \times L_{0}\right)=(t+\chi(\Gamma)) \times L_{0},
$$

which is dense in $\mathbb{R} \times L_{0}$. Hence $L$ is dense in $M$.

(iv) The leaf $\tilde{L}_{t}$ is coisotropic in $\tilde{M}$, hence has presymplectic form $\tilde{\omega}_{t}=\left.\tilde{\omega}\right|_{\tilde{L}_{t}}$, and $L_{t}$ has presymplectic form $\omega_{t}=\left.\omega\right|_{L_{t}}$. We have $\tilde{\omega}_{t}=e^{t} p^{*} \omega_{t}$, so $\Delta\left(\tilde{L}_{t}\right)=e^{t} \Delta\left(L_{t}\right)$. 
On the other hand $\Phi^{\zeta}=1$ on $M$, so $\tilde{\Phi}^{\zeta}=e^{\tilde{f}}$ on $\tilde{M}$, so $\tilde{L}_{t}=\left(\tilde{\Phi}^{\zeta}\right)^{-1}\left(e^{t}\right)$. Therefore

$$
e^{t} \Delta\left(L_{t}\right)=\Delta\left(\tilde{L}_{t}\right)=\Delta(\tilde{M}) \cap e^{t} \mathcal{H}_{\zeta}=e^{t}\left(\Delta(\tilde{M}) \cap \mathcal{H}_{\zeta}\right)=e^{t} \Delta(M)
$$

where we used Theorems 5.2 and 5.4. Hence $\Delta\left(L_{t}\right)=\Delta(M)$. The last assertion follows from Theorem 1.1.

5.9. Remarks. (i) If $\operatorname{rank}(M)=1$ the fact that $M \cong\left(\mathbb{R} \times L_{0}\right) / \Gamma$ shows that $f: M \rightarrow \mathbb{R} / \Gamma$ is the fibre bundle associated to the principal $\Gamma$-bundle $\mathbb{R} \rightarrow \mathbb{R} / \Gamma \cong \mathbb{S}^{1}$ with fibre the $\Gamma$-space $L_{0}$. The trajectories of $B$ define a flat Ehresmann connection on the bundle $M$.

(ii) For closed leaves $L$ the fact that $\Delta(L)$ is convex polyhedral follows also from the presymplectic convexity theorem of [30].

(iii) If the Lee form has zeroes, some of the leaves of $\mathcal{F}_{\theta}$ may be singular and part (ii) of the theorem may fail. Most of the other statements are however still true. Since the components of the symplectic moment map $\tilde{\Phi}$ are Morse-Bott functions, the equality $e^{\tilde{f}}=\tilde{\Phi}^{\zeta}$ implies that the potential $\tilde{f}$ is also Morse-Bott, so the Lee form $\theta$ has Morse-Bott singularities, and all its Morse indices are even. Work of Imanishi [19] and Farber $[10, \S 9.1]$ shows that the leaves of $\mathcal{F}_{\theta}$ are, locally, the level sets of $\tilde{f}$, and that the closed-dense dichotomy (iii) still holds. Part (iv) is still true for the regular leaves and presumably also for the singular leaves.

\section{Convexity For CONFORMAL SYMPleCtiC MANifolds OF THE FIRST Kind}

In this section we explore yet another relationship between conformal symplectic geometry and presymplectic geometry, which exists only for conformal symplectic manifolds of the first kind (to be defined below). The upshot is a structure theorem, Theorem 6.3, which expresses every such manifold as a generalized type of contact mapping torus, and a strong version of the conformal symplectic convexity theorem, Theorem 6.5, which is based on the presymplectic convexity theorem of [30]. One advantage of these results is that they tell us when the moment polyhedron is semirational. Another advantage is a geometric explanation for the stability of the polytopes $\Delta(L)$ for leaves $L$ of the Lee foliation: in Theorem 5.7 the fact that all leaves have the same moment polytope seems almost accidental, but for manifolds of the first kind these leaves are actually contact manifolds and there is a flow that maps them contactomorphically one onto the other, so that their moment polytopes are manifestly the same.

These facts rest on Vaisman's observation that on a conformal symplectic manifold of the first kind there exists a natural associated presymplectic form. It turns out that a group action which is Hamiltonian with respect to the conformal symplectic structure is also Hamiltonian with respect to the presymplectic structure with the same moment map. We will see that the Lee type condition corresponds to the action being clean with respect to the null foliation of the presymplectic form.

Let $M$ be a connected manifold. A conformal symplectic structure of the first kind on $M$ is a triple $(\omega, \theta, B)$, where $(\omega, \theta)$ is a conformal symplectic structure and $B$ is a vector field in $\mathfrak{X}_{\omega}(M)$ such that the function $\iota(B) \theta$ (which is constant because $L(B) \theta=0$ ) is equal to 1 . We call such a vector field $B$ an anti-Lee vector field. We say a conformal symplectic manifold of the first kind $(M, \omega, \theta, B)$ is complete if its anti-Lee vector field $B$ is complete. On a conformal symplectic manifold of the first kind the Lee form $\theta$ is nowhere 0 , so the Lee foliation $\mathcal{F}_{\theta}$ is regular. The following 
result of Vaisman states that conformal symplectic structures of the first kind are $d_{\theta}$-exact, i.e. $\omega=d_{\theta} \alpha$, and that the form $d \alpha$ is presymplectic of corank 2 .

6.1. Proposition ([38, Proposition 2.2]). Let $M$ be a connected $2 n$-dimensional manifold.

(i) Given a conformal symplectic structure of the first kind $(\omega, \theta, B)$ on $M$, the 1-form $\alpha=\iota(B) \omega$ satisfies

$$
\operatorname{rank}(d \alpha)<2 n, \quad \theta \wedge \alpha \wedge(d \alpha)^{n-1} \text { is a volume form, }
$$

and $\omega=d_{\theta} \alpha$. The Lee vector field $A$ of $(\omega, \theta)$ commutes with $B$ and is everywhere linearly independent of $B$. The form d $\alpha$ is presymplectic with null foliation $\mathcal{F}_{d \alpha}$ spanned by $A$ and $B$.

(ii) Conversely, given a 1-form $\alpha$ and a closed 1 -form $\theta$ on $M$ satisfying (6.2), the form $\omega=d_{\theta} \alpha$ is conformal symplectic of the first kind with Lee form $\theta$ and anti-Lee vector field $B$ determined by $\alpha=\iota(B) \omega$.

Contact mapping tori of higher rank. Here is a general method for producing complete conformal symplectic manifolds of the first kind. Start with a contact form $\alpha$ on a manifold $Q$. The conformal symplectic cylinder over $Q$ is the product $\tilde{M}=\mathbb{R} \times Q$ equipped with the global conformal symplectic structure $(d \alpha+d t \wedge \alpha, d t)$. The Lee vector field of the conformal symplectic cylinder is $\tilde{A}=-R$, where $R$ is the Reeb vector field of $Q$. The vector field $\tilde{B}=\partial / \partial t$ is anti-Lee. Fix $k$ commuting strict contactomorphisms, i.e. diffeomorphisms $g_{i}: Q \rightarrow Q$ satisfying $g_{i}^{*} \alpha=\alpha$ and $g_{i} \circ g_{j}=g_{j} \circ g_{i}$ for $i, j=1,2, \ldots, k$, and $k$ real numbers $c_{1}, c_{2}, \ldots, c_{k}$. The maps $\gamma_{i}: \tilde{M} \rightarrow \tilde{M}$ defined by $\gamma_{i}(t, x)=\left(t+c_{i}, g_{i}(x)\right)$ are commuting automorphisms of the conformal symplectic cylinder, which generate an action of $\Gamma=\mathbb{Z}^{k}$. We assume this $\Gamma$-action to be free and proper. (For $k=1$ this is automatically the case if $c_{1} \neq 0$. For $k \geq 2$ the action is free if $c_{1}, c_{2}, \ldots, c_{k}$ are independent over $\mathbb{Q}$, and can be proper only if $Q$ is noncompact. See Section 7 for what to do when the $\Gamma$-action is not free and proper.) The contact mapping torus of $Q$ with respect to the $g_{i}$ and the $c_{i}$ is the quotient manifold

$$
M=M_{\left(g_{1}, g_{2}, \ldots, g_{k} ; c_{1}, c_{2}, \ldots, c_{k}\right)}=\tilde{M} / \Gamma
$$

equipped with the strict conformal symplectic structure $(\omega, \theta)$ induced by $(d \alpha+$ $d t \wedge \alpha, d t)$. Proposition 6.1 shows that $(\omega, \theta)$ is of the first kind. The anti-Lee vector field $B$ of the contact mapping torus $M$ is induced by $\tilde{B}$, so $M$ is complete. The projection $\tilde{M}=\mathbb{R} \times Q \rightarrow \mathbb{R}$ induces a fibration of $M$ over the quotient $\mathbb{R} / \Gamma$, which is a genuine circle if $k=1$ and a "stacky circle" if $k \geq 2$.

Let $\tilde{\omega}=e^{\tilde{f}}(d \alpha+d t \wedge \alpha)$, where $\tilde{f}(t, x)=t$, and let $\chi: \Gamma \rightarrow \mathbb{R}$ be the inclusion. Then $(\tilde{M}, \tilde{\omega})$ is a symplectic manifold, namely the symplectization of the contact manifold $Q$, and the tuple $(\tilde{M}, \tilde{\omega}, \Gamma, \chi, \tilde{f})$ is a presentation of the contact mapping torus $M$. If the $c_{j}$ are independent over $\mathbb{Q}$, the homomorphism $\chi$ is injective, so the presentation is minimal and the rank of $M$ is equal to $k$.

The following Boothby-Wang type structure theorem, versions of which were stated in the rank 1 case by Vaisman [38, Proposition 3.3] and Bazzoni-Marrero [3, Theorem 4.6], says that every connected complete strict conformal symplectic manifold of the first kind is a contact mapping torus. 
6.3. Theorem. Let $(M, \omega, \theta, B)$ be a connected complete strict conformal symplectic manifold of the first kind. Let $(\tilde{M}, \tilde{\omega}, \Gamma, \chi, \tilde{f})$ be the minimal presentation of $(M, \omega, \theta)$.

(i) Let $\tilde{B}=p^{*} B$ and $\tilde{\alpha}=e^{\tilde{f}} p^{*} \alpha=\iota(\tilde{B}) \tilde{\omega}$. For $t \in \mathbb{R}$ let

$$
\tilde{Q}_{t}=\tilde{f}^{-1}(t), \quad \tilde{\alpha}_{t}=\left.\tilde{\alpha}\right|_{\tilde{Q}_{t}}, \quad Q_{t}=p\left(\tilde{Q}_{t}\right), \quad \alpha_{t}=\left.\alpha\right|_{Q_{t}} .
$$

Then $\left(\tilde{Q}_{t}, \tilde{\alpha}_{t}\right)$ and $\left(Q_{t}, \alpha_{t}\right)$ are contact manifolds for all $t$. The Reeb vector field on $Q_{t}$ is equal to $-A$, where $A$ is the Lee vector field of $M$. The flow of $B$ induces strict contactomorphisms $Q_{0} \rightarrow Q_{t}$ for all $t$.

(ii) We have $F_{0}^{*} \tilde{\omega}=e^{t}\left(d \alpha_{0}+d t \wedge \alpha_{0}\right)$, where $F_{0}: \mathbb{R} \times Q_{0} \rightarrow \tilde{M}$ is the diffeomorphism of Theorem 5.7 (ii). It follows that $(\tilde{M}, \tilde{\omega})$ is the symplectization of $\left(Q_{0}, \alpha_{0}\right) \cong\left(\tilde{Q}_{0}, \tilde{\alpha}_{0}\right)$.

(iii) We have $\left(p \circ F_{0}\right)^{*} \omega=d \alpha_{0}+d t \wedge \alpha_{0}$. It follows that $\left(\tilde{M}, p^{*} \omega\right) \cong(\mathbb{R} \times$ $\left.Q_{0}, d \alpha_{0}+d t \wedge \alpha_{0}, d t\right)$ is the conformal symplectic cylinder over $Q_{0}$ and that its quotient by the $\Gamma$-action $(M, \omega, \theta)$ is a contact mapping torus.

Proof. (i) Let $\operatorname{dim}(M)=2 n$. It follows from Proposition 6.1 that

$$
\alpha \wedge(d \alpha)^{n-1}=\alpha \wedge(\omega-\theta \wedge \alpha)^{n-1}=\alpha \wedge \omega^{n-1} .
$$

Now $\theta \wedge \alpha \wedge \omega^{n-1}$ is a volume form on $M$, so $\alpha \wedge(d \alpha)^{n-1}$ restricts to a volume form on $Q_{t}$, so $\alpha_{t}$ is a contact form. We have $\tilde{\alpha}_{t}=e^{t} p^{*} \alpha_{t}$, so $\tilde{\alpha}_{t}$ is contact as well. Also

$$
\begin{aligned}
\iota(A) \alpha & =-\iota(B) \iota(A) \omega=-\iota(B) \theta=-1, \\
\iota(A) d \alpha & =\iota(A)(\omega-\theta \wedge \alpha)=\theta+(\iota(A) \alpha) \theta=0,
\end{aligned}
$$

so $-A$ is the Reeb vector field of $\alpha$. Theorem 5.7(ii) says that the time $t$ flow of $B$ maps $Q_{0}$ to $Q_{t}$. From $L(B) \omega=0$ we get $L(B) \alpha=L(B) \iota(B) \omega=-\iota(B) L(B) \omega=0$, so the flow of $B$ preserves $\alpha$.

(ii)-(iii) Using $\omega=d_{\theta} \omega$ (Proposition 6.1$), F_{0}^{*} p^{*} \alpha=\alpha_{0}$, and $F_{0}^{*} \tilde{f}=t$ gives

$$
F_{0}^{*} p^{*} \omega=F_{0}^{*} p^{*}(d \alpha+\theta \wedge \alpha)=d \alpha_{0}+d t \wedge \alpha_{0} .
$$

Hence also $F_{0}^{*} \tilde{\omega}=F_{0}^{*}\left(e^{\tilde{f}} p^{*} \omega\right)=e^{t}\left(d \alpha_{0}+d t \wedge \alpha_{0}\right)$.

In the presence of a Hamiltonian action we have the following additional information.

6.4. Proposition. Let $(M, \omega, \theta, B)$ be a connected strict conformal symplectic manifold of the first kind which is equipped with a Hamiltonian $K$-action with moment $\operatorname{map} \Phi: M \rightarrow \mathfrak{k}^{*}$.

(i) We may assume the anti-Lee vector field $B$ to be $K$-invariant. For such a choice of $B$ we have $L(B) \Phi^{\xi}=0$ for all $\xi \in \mathfrak{k}$.

(ii) Suppose $B$ is $K$-invariant and let $\alpha=\iota(B) \omega$. The $K$-action is Hamiltonian with respect to the presymplectic form $d \alpha$ and with the same moment map $\Phi$.

(iii) Suppose the $K$-action on $(M, \omega, \theta)$ is of Lee type and let $\zeta \in \mathfrak{z}(\mathfrak{k})$ be a Lee element. Then the $K$-action on $\left(M, \mathcal{F}_{d \alpha}\right)$ is clean with null ideal $\mathbb{R} \zeta \oplus \mathfrak{n}$, where $\mathfrak{n}=\operatorname{ker}(\mathfrak{k} \rightarrow \mathfrak{X}(M))$ is the kernel of the infinitesimal action.

(iv) Suppose $B$ is $K$-invariant and complete. Let $(\tilde{M}, \tilde{\omega}, \Gamma, \chi, \tilde{f})$ be the minimal presentation of $(M, \omega, \theta)$, and let $Q_{0}$ and $F_{0}: \mathbb{R} \times Q_{0} \rightarrow \tilde{M}$ be as in Theorem 6.3. Let $\Psi: Q_{0} \rightarrow \mathfrak{k}^{*}$ be the contact moment map for the $K$ action on $Q_{0}$ defined by $\Psi^{\xi}=-\iota\left(\xi_{Q_{0}}\right) \alpha_{0}$ for $\xi \in \mathfrak{k}$. Then the moment 
map for the $K$-action on the conformal symplectic cylinder $\left(\tilde{M}, p^{*} \omega\right) \cong$ $\left(\mathbb{R} \times Q_{0}, d \alpha_{0}+d t \wedge \alpha_{0}, d t\right)$ is given by

$$
\left(p \circ F_{0}\right)^{*} \Phi(t, x)=\Psi(x),
$$

and the moment map for the $K$-action on the symplectic cone $(\tilde{M}, \tilde{\omega}) \cong$ $\left(\mathbb{R} \times Q_{0}, e^{t}\left(d \alpha_{0}+d t \wedge \alpha_{0}\right)\right)$ is given by

$$
F_{0}^{*} \tilde{\Phi}(t, x)=e^{t} \Psi(x) .
$$

Proof. (i) Let $\bar{B}=\int_{K} g_{*} B d g$. Using the $K$-invariance of $\omega$ and $\theta$ (Proposition 3.2) we get

$$
\iota(\bar{B}) \theta=\int_{K} \iota\left(g_{*} B\right) \theta d g=\int_{K} g^{*}(\iota(B) \theta) d g=\int_{K} g^{*}(1) d g=1,
$$

and similarly $L(\bar{B}) \omega=0$, so $\bar{B}$ is anti-Lee and $K$-invariant. Let $\xi \in \mathfrak{k}$ and suppose $B$ is $K$-invariant. Then $\left[\xi_{M}, B\right]=0$, so $L_{\theta}(B) \iota\left(\xi_{M}\right)=\iota\left(\xi_{M}\right) L_{\theta}(B)$ by (2.2). Using $d_{\theta} \Phi^{\xi}=\iota\left(\xi_{M}\right) \omega$ yields

$$
\begin{aligned}
d_{\theta} L_{\theta}(B) \Phi^{\xi} & =L_{\theta}(B) d_{\theta} \Phi^{\xi}=L_{\theta}(B) \iota\left(\xi_{M}\right) \omega=\iota\left(\xi_{M}\right) L_{\theta}(B) \omega \\
& =\iota\left(\xi_{M}\right)(L(B) \omega+\theta(B) \omega)=\iota\left(\xi_{M}\right) \omega=d_{\theta} \Phi^{\xi},
\end{aligned}
$$

so $L_{\theta}(B) \Phi^{\xi}=\Phi^{\xi}$ by injectivity of $d_{\theta}$ (Proposition 2.4). Hence $L(B) \Phi^{\xi}=0$.

(ii) We must show that $d \Phi^{\xi}=\iota\left(\xi_{M}\right) d \alpha$ for all $\xi \in \mathfrak{k}$. Proposition 6.1(i) gives $\omega=d_{\theta} \alpha$. Using $d_{\theta} \Phi^{\xi}=\iota\left(\xi_{M}\right) \omega$ and $L_{\theta}\left(\xi_{M}\right)=\left[d_{\theta}, \iota\left(\xi_{M}\right)\right]$ (see (2.2)) we get

$$
d_{\theta} \Phi^{\xi}=\iota\left(\xi_{M}\right) d_{\theta} \alpha=L_{\theta}\left(\xi_{M}\right) \alpha-d_{\theta} \iota\left(\xi_{M}\right) \alpha=L\left(\xi_{M}\right) \alpha+\theta\left(\xi_{M}\right) \cdot \alpha-d_{\theta} \iota\left(\xi_{M}\right) \alpha .
$$

Since $B$ and $\omega$ are invariant, $\alpha=\iota(B) \omega$ is invariant, so $L\left(\xi_{M}\right) \alpha=0$. Since $\iota\left(\xi_{M}\right) \theta=0$ (Proposition 3.2) and $L\left(\xi_{M}\right) \alpha=0$, this gives $d_{\theta} \Phi^{\xi}=-d_{\theta}\left(\iota\left(\xi_{M}\right) \alpha\right)$. By Proposition 2.4, $d_{\theta}: \mathcal{C}^{\infty}(M) \rightarrow \Omega^{1}(M)$ is injective, so $\Phi^{\xi}=-\iota\left(\xi_{M}\right) \alpha$ and thus $d \Phi^{\xi}=-d \iota\left(\xi_{M}\right) \alpha=\iota\left(\xi_{M}\right) d \alpha$.

(iii) Let $\xi \in \mathfrak{k}$ and suppose $\xi_{M}$ is tangent to the null foliation $\mathcal{F}_{d \alpha}$, which according to Proposition 6.1 is spanned by $A=\zeta_{M}$ and $B$. Then $\xi_{M}=\lambda \zeta_{M}+\mu B$ for some $\lambda$ and $\mu$. Using $\iota\left(\xi_{M}\right) \theta=\iota\left(\zeta_{M}\right) \theta=0$ and $\iota(B) \theta=1$ we obtain $\xi_{M}=\lambda \zeta_{M}$, so $\xi \in \mathbb{R} \zeta \oplus \mathfrak{n}$. This shows that the null ideal is equal to $\mathbb{R} \zeta \oplus \mathfrak{n}$ and that

$$
T_{m}(K \cdot m) \cap T_{m} \mathcal{F}=\mathbb{R} \zeta_{M}(m)=T_{m}(N \cdot m)
$$

for all $m \in M$.

(iv) Let $\xi \in \mathfrak{k}$. Since $\alpha_{0}$ and $\xi_{Q_{0}}$ are $\Gamma$-invariant, the function $\Psi^{\xi}$ is $\Gamma$-invariant and therefore descends to a function $\psi^{\xi}$ on $M$. It follows from (i) that

$$
\psi^{\xi}=-\iota\left(\xi_{M}\right) \alpha=-\iota\left(\xi_{M}\right) \iota(B) \omega=\iota(B) \iota\left(\xi_{M}\right) \omega=\iota(B) d_{\theta} \Phi^{\xi}=L_{\theta}(B) \Phi^{\xi}=\Phi^{\xi},
$$

which proves the first statement. The second statement follows immediately from the first.

Combining Theorem 6.3 and Proposition 6.4 with the presymplectic convexity theorem [30, Theorem 2.2] yields the following result.

6.5. Theorem. Let $(M, \omega, \theta, B)$ be a connected strict conformal symplectic manifold of the first kind equipped with a Hamiltonian $K$-action with moment map $\Phi: M \rightarrow$ $\mathfrak{k}^{*}$ and with an anti-Lee vector field $B$ that is $K$-invariant and complete. Let $T$ be a maximal torus of $K$ and $\mathfrak{t}=\operatorname{Lie}(T)$. Choose a closed Weyl chamber $C$ in $\mathfrak{t}^{*}$ and define $\Delta(M)=\Phi(M) \cap C$. Suppose the $K$-action is of Lee type with central Lee element $\zeta \in \mathfrak{k}$. 
(i) All leaves $Q$ of the Lee foliation $\mathcal{F}_{\theta}$ of $M$ are equivariantly contactomorphic to one another. We have $\Delta(Q)=\Delta(M)$ for every $Q$, and $\Delta(\tilde{M})=$ $\mathbb{R}_{>0} \cdot \Delta(M)$ and $\Delta(\tilde{M})=\Delta(M) \cap \mathcal{H}_{\zeta}$ for every presentation $\tilde{M}$ of $M$.

(ii) Suppose $\Phi$ is proper. Then $\Phi: M \rightarrow \Phi(M)$ is an open map with connected fibres; $\Delta(M)$ is a closed convex polyhedral set; $\Delta(\tilde{M}) \cup\{0\}$ is a convex polyhedral cone; and $\Delta(M)$ is semirational if and only if the line $\mathbb{R} \zeta$ in $\mathfrak{t}$ is rational.

\section{EXAMPLES}

Cotangent bundles. Here is an example of a Hamiltonian action on a strict conformal symplectic manifold which is not of Lee type, but where the conclusion of the convexity theorem nevertheless holds. Let $Q$ be a connected $K$-manifold with $H_{\text {bas }}^{1}(Q) \neq 0$. Let $M=T^{*} Q$ be its cotangent bundle and $\pi: M \rightarrow Q$ the projection. The $K$-action on $Q$ lifts naturally to a $K$-action on $M$. Let $\alpha \in \Omega^{1}(M)$ be the tautological 1-form; then $\omega_{0}=d \alpha$ is the standard symplectic form on $M$. We modify $\omega_{0}$ as follows: we choose a closed but not exact basic 1-form $\theta_{Q} \in \Omega_{\text {bas }}^{1}(Q)$, and we put $\theta=\pi^{*} \theta_{Q}$ and $\omega=d_{\theta} \alpha$. Then $(\omega, \theta)$ is a $K$-invariant strict conformal symplectic structure on $M$. For $\xi \in \mathfrak{k}$ define a function $\Phi^{\xi}$ on $M$ by $\Phi^{\xi}=-\iota\left(\xi_{M}\right) \alpha$; then

$$
d_{\theta} \Phi^{\xi}=-L_{\theta}\left(\xi_{M}\right) \alpha+\iota\left(\xi_{M}\right) d_{\theta} \alpha=\iota\left(\xi_{M}\right) \omega
$$

because $\theta$ is basic. So the action is Hamiltonian with moment map $\Phi$. However, the action is not of Lee type. If there existed a Lee element $\zeta \in \mathfrak{k}$, we would have $1=\Phi^{\zeta}=-\iota\left(\zeta_{M}\right) \alpha$, i.e. $\beta\left(\zeta_{M}\right)=-1$ for all $q \in Q$ and $\beta \in T_{q}^{*} Q$. Taking $\beta=\left(\theta_{Q}\right)_{q}$ and using that $\theta_{Q}$ is basic gives $0=\left(\theta_{Q}\right)_{q}\left(\zeta_{M}\right)=-1$, a contradiction. Nevertheless the intersection $\Phi(M) \cap C$ is convex, indeed a rational convex polyhedral cone. This follows from the fact that $\Phi$ is also the moment map for the standard symplectic form $\omega_{0}$ and from the symplectic convexity theorem for cotangent bundles, [36, Theorem 7.6].

This example prompts the question whether the Lee type hypothesis of Theorem 1.1 can be weakened. Let $(M, \omega, \theta)$ be a strict conformal symplectic manifold equipped with a Hamiltonian $K$-action. Let $A$ be the Lee vector field and let $\mathcal{F}_{A}$ be the (possibly singular) 1-dimensional foliation of $M$ generated by $A$. Define

$$
\mathfrak{n}_{A}=\left\{\xi \in \mathfrak{k} \mid \xi_{M} \text { is tangent to } \mathcal{F}_{A}\right\} ;
$$

then $\mathfrak{n}_{A}$ is an ideal of $\mathfrak{k}$. Let $N_{A}$ be the normal subgroup of $K$ with Lie algebra $\mathfrak{n}_{A}$. Then $N_{A} \cdot x \subset K \cdot x \cap \mathcal{F}_{A}(x)$ for all $x \in M$, where $\mathcal{F}_{A}(x)$ denotes the $\mathcal{F}_{A}$-leaf of $x$, and hence $T_{x}\left(N_{A} \cdot x\right) \subset T_{x}(K \cdot x) \cap T_{x} \mathcal{F}_{A}$. If the action is of Lee type with Lee element $\zeta$ we have $\mathfrak{n}_{A}=\mathfrak{n}+\mathbb{R} \zeta$, where $\mathfrak{n}$ is the kernel of the infinitesimal action $\mathfrak{k} \rightarrow \mathfrak{X}(M)$, and therefore

$$
T_{x}\left(N_{A} \cdot x\right)=T_{x}(K \cdot x) \cap T_{x} \mathcal{F}_{A}
$$

for all $x$. In the cotangent example above the Lee vector field is tangent to the fibres of $\pi: M \rightarrow Q$, so we have $T_{x}(K \cdot x) \cap T_{x} \mathcal{F}_{A}=0$ for all $x$, and therefore (7.1) is satisfied as well.

7.2. Problem. Suppose the K-action on $M$ satisfies the "cleanness" condition (7.1) and that the moment map $\Phi: M \rightarrow \mathfrak{k}^{*}$ is proper. Do the conclusions of the convexity theorem, Theorem 1.1, hold for $M$ ? 


\section{Contact mapping tori of rank 1.}

Contact ellipsoids. Let $c>1$ and let $h: \mathbb{C}^{n} \rightarrow \mathbb{C}^{n}$ be the homothety $h(z)=c z$. Then $h^{*} \tilde{\omega}=c^{2} \tilde{\omega}$, where $\tilde{\omega}=\frac{i}{2} \sum d z_{j} \wedge d \bar{z}_{j}$ is the standard symplectic form. Choose numbers $\zeta_{1} \geq \zeta_{2} \geq \cdots \geq \zeta_{n}>0$ and let $\tilde{f}(z)=\log \left(\frac{1}{2} \sum_{j} \zeta_{j}\left|z_{j}\right|^{2}\right)$. Then $\tilde{f}(h(z))=$ $\tilde{f}(z)+2 \log c$. The pair $\left(e^{-\tilde{f}} \tilde{\omega}, d \tilde{f}\right)$ defines a global conformal symplectic structure on $\tilde{M}=\mathbb{C}^{n} \backslash\{0\}$, which is invariant under the cyclic group $\Gamma$ generated by $h$. This group acts properly and freely on $\tilde{M}$ because $c>1$, so the quotient $M=\tilde{M} / \Gamma$ is a conformal symplectic manifold called a Hopf manifold. As a conformal symplectic manifold $M$ is the trivial contact mapping torus obtained from the contact ellipsoid

$$
Q=Q_{\zeta}=\tilde{f}^{-1}(0)=\left\{\left.z \in \mathbb{C}^{n}\left|\frac{1}{2} \sum_{j} \zeta_{j}\right| z_{j}\right|^{2}=1\right\}
$$

and the identity automorphism $Q \rightarrow Q$. As a manifold

$$
M=\mathbb{S}^{1} \times \mathbb{S}^{2 n-1}
$$

and therefore its second Betti number vanishes. Hence it does not admit any symplectic, let alone Kähler, structures, although it admits many complex structures. The minimal presentation of $M$ is $(\tilde{M}, \tilde{\omega}, \Gamma, \chi, \tilde{f})$, where $\chi: \Gamma \rightarrow \mathbb{R}$ is defined by $\chi\left(h^{n}\right)=2 n \log c$.

We equip $\mathfrak{u}(n)=\operatorname{Lie}(\mathbf{U}(n))$, the Lie algebra of anti-Hermitian matrices, with the trace form $\langle\xi, \eta\rangle=-\operatorname{tr}(\xi \eta)$, and use this to identify $\mathfrak{u}(n)^{*} \cong \mathfrak{u}(n)$. The standard action of the unitary group $\mathbf{U}(n)$ on $\mathbb{C}^{n}$ commutes with the $\Gamma$-action and is Hamiltonian relative to $\tilde{\omega}$ with moment map given by $\tilde{\Phi}(z)=\frac{i}{2} z z^{*}$. Here we think of $z$ as a column vector and denote by $z^{*}$ its conjugate transpose. Let $K$ be a closed subgroup of $\mathbf{U}(n)$ whose Lie algebra $\mathfrak{k}$ contains $\zeta=i \operatorname{diag}\left(\zeta_{1}, \zeta_{2}, \ldots, \zeta_{n}\right)$ as a central element. (If the $\zeta_{j}$ are pairwise distinct, this forces $K$ to be the diagonal maximal torus of $\mathbf{U}(n)$.) The $K$-action is Hamiltonian with moment map given by $\tilde{\Phi}^{\xi}(z)=-\frac{i}{2} z^{*} \xi z$ for $\xi \in \mathfrak{k}$ and $z \in \mathbb{C}^{n}$. We have $e^{\tilde{f}}=\tilde{\Phi}^{\zeta}$, so the induced $K$-action on $M$ is Hamiltonian with moment map

$$
\Phi^{\xi}([z])=e^{-\tilde{f}(z)} \tilde{\Phi}^{\xi}(z)=\frac{z^{*} \xi z}{z^{*} \zeta z}
$$

and is of Lee type with central Lee element $\zeta$. The moment map $\tilde{\Phi}$ is proper (because $\tilde{f}$ is proper) and the moment body $\Delta(\tilde{M})$ is the rational convex polyhedral cone spanned by the highest weights of the irreducible $K$-modules occurring in the symmetric algebra of $\mathbb{C}^{n}([\underset{\sim}{36}$, Theorem 4.9]). The moment body $\Delta(M)=\Delta(Q)$ is the convex polytope $\Delta(\tilde{M}) \cap \mathcal{H}_{\zeta}$. It is semirational if and only if the $\mathbb{Q}$-linear subspace of $\mathbb{R}$ spanned by $\zeta_{1}, \zeta_{2}, \ldots, \zeta_{n}$ is one-dimensional.

Contact hyperboloids. Fix numbers $\zeta_{1} \geq \zeta_{2} \geq \cdots \geq \zeta_{n}>0$ and let $\zeta$ be the diagonal matrix $\operatorname{diag}\left(\zeta_{1}, \zeta_{2}, \ldots, \zeta_{n}\right)$. Write vectors in $\mathbb{R}^{2 n}$ as pairs $(x, y) \in \mathbb{R}^{n} \times \mathbb{R}^{n}$ and define the split quadratic form $q=q_{\zeta}$ on $\mathbb{R}^{2 n}$ by $q(x, y)=x^{\top} \zeta y=\sum_{j} \zeta_{j} x_{j} y_{j}$, where $x^{\top}$ denotes the transpose of the column vector $x$. Let

$$
\tilde{M}=\left\{(x, y) \in \mathbb{R}^{2 n} \mid q(x, y)>0\right\}
$$

and define $\tilde{f}: \tilde{M} \rightarrow \mathbb{R}$ by $\tilde{f}(x, y)=\log q(x, y)$. Let $\tilde{\omega}=\sum_{j} d x_{j} \wedge d y_{j}$ be the standard symplectic form. Then $\left(\tilde{M}, e^{-\tilde{f}} \tilde{\omega}, d \tilde{f}, \tilde{B}\right)$ is a conformal symplectic manifold of the 
first kind with Lee and anti-Lee vector fields given by

$$
\tilde{A}=\sum_{j} \zeta_{j}\left(x_{j} \frac{\partial}{\partial x_{j}}-y_{j} \frac{\partial}{\partial y_{j}}\right), \quad \tilde{B}=\frac{1}{2} \sum_{j}\left(x_{j} \frac{\partial}{\partial x_{j}}+y_{j} \frac{\partial}{\partial y_{j}}\right) .
$$

By Theorem $6.3 \tilde{M}$ is the conformal symplectic cylinder over the contact "hyperboloid"

$$
Q=Q_{\zeta}=\tilde{f}^{-1}(0)=\left\{(x, y) \in \mathbb{R}^{2 n} \mid q(x, y)=1\right\}
$$

which is equipped with the contact form $\left.\iota(\tilde{B}) \tilde{\omega}\right|_{Q}=\left.\frac{1}{2} \sum_{j}\left(x_{j} d y_{j}-y_{j} d x_{j}\right)\right|_{Q}$.

Recall that $\mathfrak{s p}(2 n, \mathbb{R})$, the Lie algebra of the symplectic group $\mathbf{S p}(2 n, \mathbb{R})$, consists of all block matrices $\left(\begin{array}{ll}a_{11} & a_{12} \\ a_{21} & a_{22}\end{array}\right)$ with blocks $a_{i j} \in \mathfrak{g l}(n, \mathbb{R})$ satisfying

$$
a_{12}=a_{12}^{\top}, \quad a_{21}=a_{21}^{\top}, \quad a_{22}=-a_{11}^{\top} .
$$

Similarly, the orthogonal group $\mathbf{O}(\zeta)$ of the quadratic form $q_{\zeta}$ has Lie algebra $\mathfrak{o}(\zeta)$ consisting of all block matrices $\left(\begin{array}{ll}a_{11} & a_{12} \\ a_{21} & a_{22}\end{array}\right)$ with blocks $a_{i j} \in \mathfrak{g l}(n, \mathbb{R})$ satisfying

$$
a_{12}=-\zeta^{-1} a_{12}^{\top} \zeta, \quad a_{21}^{\top}=-\zeta^{-1} a_{21} \zeta, \quad a_{22}=-\zeta^{-1} a_{11}^{\top} \zeta
$$

Let $\mathfrak{g}=\{\eta \in \mathfrak{g l}(n, \mathbb{R}) \mid[\eta, \zeta]=0\}$ be the centralizer of $\zeta \in \mathfrak{g l}(n, \mathbb{R})$ and $G$ the closed connected subgroup of $\mathbf{G L}(n, \mathbb{R})$ with Lie algebra $\mathfrak{g}$. The map $\mathfrak{g l}(n, \mathbb{R}) \rightarrow \mathfrak{g l}(2 n, \mathbb{R})$ defined by $\eta \mapsto\left(\begin{array}{cc}\eta & 0 \\ 0 & -\eta^{\top}\end{array}\right)$ is an isomorphism

$$
\mathfrak{g} \stackrel{\cong}{\longrightarrow} \mathfrak{p}(2 n, \mathbb{R}) \cap \mathfrak{o}(\zeta)
$$

which we will use to identify $\mathfrak{g}$ with $\mathfrak{s p}(2 n, \mathbb{R}) \cap \mathfrak{o}(\zeta)$ and $G$ with the identity component of $\mathbf{S p}(2 n, \mathbb{R}) \cap \mathbf{O}(\zeta)$. Thus $G$ acts on $\mathbb{R}^{2 n}=T^{*} \mathbb{R}^{n}$ by the lifted cotangent action $g \cdot(x, y)=\left(g x,\left(g^{-1}\right)^{\top} y\right)$, which preserves both the symplectic form $\tilde{\omega}$ and the quadratic form $q_{\zeta}$. A split Cartan subalgebra of $\mathfrak{g}$ is

$$
\mathfrak{h}=\left\{\eta=\operatorname{diag}\left(\eta_{1}, \eta_{2}, \ldots, \eta_{n}\right) \mid \eta_{1}, \eta_{2}, \ldots, \eta_{n} \in \mathbb{R}\right\}
$$

Observe that the Lee vector field is in $\mathfrak{h}$, namely $\tilde{A}=\zeta$. Let $H$ be the Cartan subgroup of $G$ with Lie algebra $\mathfrak{h}$. Choose $\eta=\operatorname{diag}\left(\eta_{1}, \eta_{2}, \ldots, \eta_{n}\right) \in \mathfrak{h}$ and put

$$
h_{0}=\exp \zeta, \quad h_{1}=\exp \eta \in H
$$

These elements generate subgroups $\left\langle h_{0}\right\rangle$ and $\left\langle h_{1}\right\rangle$ of $H$, both of which act properly on $Q$, but the subgroup $\left\langle h_{0}, h_{1}\right\rangle \cong \mathbb{Z}^{2}$ does not act properly. However, choose $c \neq 0$ and define $\gamma_{0}, \gamma_{1}: \tilde{M} \rightarrow \tilde{M}$ by

$$
\gamma_{0}(x, y)=h_{0} \cdot(x, y), \quad \gamma_{1}(x, y)=e^{\frac{1}{2} c} h_{1} \cdot(x, y) .
$$

Then $\Gamma=\left\langle\gamma_{0}, \gamma_{1}\right\rangle \cong \mathbb{Z}^{2}$ acts properly and freely on $\tilde{M}$. We have $\gamma_{i}^{*} \tilde{\omega}=e^{c_{i}} \tilde{\omega}$ and $\gamma_{i}^{*} \tilde{f}=\tilde{f}+c_{i}$, where $c_{0}=0$ and $c_{1}=c$, so the quotient $M=\tilde{M} / \Gamma$ is a conformal symplectic manifold with presentation $(\tilde{M}, \tilde{\omega}, \Gamma, \chi, \tilde{f})$, where $\chi: \Gamma \rightarrow \mathbb{R}$ is defined by $\chi\left(\gamma_{i}\right)=c_{i}$. Since $\operatorname{ker}(\chi)=\left\langle\gamma_{0}\right\rangle$, the minimal presentation of $M$ is $\tilde{M} /\left\langle\gamma_{0}\right\rangle$ and the rank of $M$ is 1 . As a conformal symplectic manifold $M$ is the contact mapping torus of the contact manifold $Q /\left\langle h_{0}\right\rangle$ with respect to the contact automorphism $h_{1}: Q /\left\langle h_{0}\right\rangle \rightarrow Q /\left\langle h_{0}\right\rangle$. The Lee vector field of $M$ is $A=p_{*} \tilde{A}$, which is periodic since $\exp \tilde{A}=\gamma_{0}$. To compute the structure of $M$ as a manifold we may assume that $\zeta_{1}=\zeta_{2}=\cdots=\zeta_{n}=1$, in which case $G$ is equal to $\mathbf{G L}_{0}(n, \mathbb{R})$, the 
identity component of $\mathbf{G L}(n, \mathbb{R})$, which acts transitively on $Q$. Using the Mostow decomposition theorem for homogeneous spaces we get

$$
\begin{aligned}
Q & \cong \mathbf{G L}_{0}(n, \mathbb{R}) / \mathbf{G L}_{0}(n-1, \mathbb{R}) \cong\left(\mathbf{S O}(n) \times \mathbb{R}^{n}\right) / \mathbf{S O}(n-1) \\
& \cong \mathbb{R} \times\left(\mathbf{S O}(n) \times \mathbb{R}^{n-1}\right) / \mathbf{S O}(n-1) \cong \mathbb{R} \times T^{*} \mathbb{S}^{n-1},
\end{aligned}
$$

and therefore

$$
M \cong \mathbb{S}^{1} \times \mathbb{S}^{1} \times T^{*} \mathbb{S}^{n-1} .
$$

Next we display some Hamiltonian actions of Lee type on $M$. A maximal compact subgroup of $G$ is

$$
G_{0}=G \cap \mathbf{S O}(n),
$$

which is the identity component of $\{g \in \mathbf{S O}(n) \mid[g, \zeta]=0\}$. Let $K_{0}$ be any closed connected subgroup of $G_{0}$ which centralizes $h_{1}$ and let $\tilde{K}$ be the closed connected subgroup $K_{0} \exp (\mathbb{R} \zeta)$ of $G_{0}$. Then the $\tilde{K}$-action on $\tilde{M}$ descends to a $\tilde{K}$-action on $M=\tilde{M} / \Gamma$. The subgroup $\left\langle h_{0}\right\rangle$ of $\tilde{K}$ acts trivially on $M$, so we get an action of the compact Lie group

$$
K=\tilde{K} /\left\langle h_{0}\right\rangle \cong \exp (\mathbb{R} \zeta) / \exp (\mathbb{Z} \zeta) \times K_{0} \cong \mathbb{S}^{1} \times K_{0}
$$

on $M$, which is Hamiltonian and of Lee type with central Lee element $\zeta$. The standard action of $\mathbf{S O}(n)$ on $\mathbb{R}^{2 n}$ is Hamiltonian relative to $\tilde{\omega}$ with moment map given by $\tilde{\Phi}(x, y)=x y^{\top}-y x^{\top}$. The contact manifold $Q$ is not compact and the moment map for the $K_{0}$-action on $Q$ is not proper (unless $K_{0}=\mathbf{S O}(n)$ ), but nevertheless the moment body $\Delta_{0}(Q)$ for the $K_{0}$-action is convex, in fact a rational convex polyhedral cone, by Theorem A.2 and Example A.3 below. Therefore the moment body for the $K$-action is $\Delta(Q)=\{1\} \times \Delta_{0}(Q) \subseteq \mathbb{R} \times \mathfrak{k}_{0}^{*}$, and it follows from Theorem 6.5 that $\Delta(M)=\Delta(Q)$.

Contact mapping tori of rank $\geq 2$. Higher rank contact mapping tori with interesting group actions are harder to come by. Below is a slightly contrived class of examples, which is based on the simple observation that the product $P \times Q$ of an exact symplectic manifold $\left(P, \omega_{P}=d \alpha_{P}\right)$ and a contact manifold $\left(Q, \alpha_{Q}\right)$ is a contact manifold with contact form $\alpha=\operatorname{pr}_{P}^{*} \alpha_{P}+\operatorname{pr}_{Q}^{*} \alpha_{Q}$.

Suppose we are given $k$ commuting strict contactomorphisms $g_{i}: Q \rightarrow Q$. These generate a strict contact action of $\Gamma=\mathbb{Z}^{k}$, which is not necessarily free or proper. Now let us take $P=T^{*} \mathbb{R}^{k}$ with its standard 1-form $\alpha_{0}=-\sum_{j} y_{j} d x_{j}$ and standard symplectic structure $\omega_{0}=d \alpha_{0}$ and form the contact manifold $T^{*} \mathbb{R}^{k} \times Q$. Then $\Gamma=\mathbb{Z}^{k}$ acts freely and properly on $\mathbb{R}^{k}$ by translations, and the cotangent lift of this action to $T^{*} \mathbb{R}^{k}$ preserves $\alpha_{0}$. Therefore the diagonal $\Gamma$-action on $T^{*} \mathbb{R}^{k} \times Q$ is a proper and free strict contact action. This allows us for any choice of real numbers $c_{1}, c_{2}, \ldots, c_{k}$ to form the contact mapping torus of $T^{*} \mathbb{R}^{k} \times Q$,

$$
\widehat{M}=\widehat{M}_{\left(g_{1}, g_{2}, \ldots, g_{k} ; c_{1}, c_{2}, \ldots, c_{k}\right)}=\left(\mathbb{R} \times T^{*} \mathbb{R}^{k} \times Q\right) / \Gamma,
$$

which we will call the fattened contact mapping torus of $Q$ with respect to the $g_{i}$ and the $c_{i}$. Write points of $\mathbb{R} \times T^{*} \mathbb{R}^{k} \times Q$ as quadruplets $(t, x, y, q)$ with $t \in \mathbb{R}$, $x, y \in \mathbb{R}^{k}$ and $q \in Q$. Let $\tilde{f}(t, x, y, q)=t$ and

$$
\tilde{\omega}=e^{t}\left(d\left(\alpha_{0}+\alpha_{Q}\right)+d t \wedge\left(\alpha_{0}+\alpha_{Q}\right)\right) .
$$

Also define $\chi: \Gamma \rightarrow \mathbb{R}$ by $\chi\left(\gamma_{i}\right)=c_{i}$, where the $\gamma_{i}$ are the standard generators of $\Gamma$. Then $\left(\mathbb{R} \times T^{*} \mathbb{R}^{k} \times Q, \tilde{\omega}, \Gamma, \chi, \tilde{f}\right)$ is a presentation of $\widehat{M}$. If the $c_{i}$ are independent over $\mathbb{Q}$, this presentation is minimal and $\widehat{M}$ has rank $k$. 
The fattened mapping torus $\widehat{M}$ relates to the usual mapping torus $M$ as follows. The left translation action of $\mathbb{R}^{k}$ on $\mathbb{R} \times T^{*} \mathbb{R}^{k} \times Q$ defined by $u \cdot(t, x, y, q)=$ $(t, x+u, y, q)$ descends to a Hamiltonian $\mathbb{R}^{k}$-action on $\widehat{M}$ whose moment map is linear momentum $\mu([t, x, y, q])=y$. The reduced space of $\widehat{M}$ with respect to $\mathbb{R}^{k}$ is

$$
\widehat{M} / / \mathbb{R}^{k}=M=M_{\left(g_{1}, g_{2}, \ldots, g_{k} ; c_{1}, c_{2}, \ldots, c_{k}\right)} .
$$

The reduced space $M$ exists as a manifold if and only if the $\Gamma$-action on $\mathbb{R} \times Q$ is free and proper. If $M$ is not a manifold, we can interpret it as a conformal symplectic stack in the spirit of [18]: the zero fibre $\mu^{-1}(0)=\left(\mathbb{R} \times \mathbb{R}^{k} \times Q\right) / \Gamma$ is a conformal presymplectic manifold in the sense of Appendix B, and the quotient map $\mu^{-1}(0) \rightarrow M$ is an atlas of the stack $M$.

Suppose $K$ acts on $Q$ by strict contactomorphisms. Such an action is Hamiltonian with moment map $\Psi: Q \rightarrow \mathfrak{k}^{*}$ given by $\Psi^{\xi}(q)=-\iota\left(\xi_{Q}\right) \alpha_{Q}$. Supposing that the $K$-action commutes with the $\Gamma$-action, we get a Hamiltonian $K$-action on the fattened contact mapping torus defined by $k \cdot[t, x, y, q]=[t, x, y, k \cdot q]$. The moment map $\widehat{\Phi}: \widehat{M} \rightarrow \mathfrak{k}^{*}$ is given by $\widehat{\Phi}([t, x, y, q])=\Psi(q)$ and the moment body $\Delta(\widehat{M})=\widehat{\Phi}(\widehat{M}) \cap C$ is equal to $\Delta(Q)$.

Examples of contact mapping tori of arbitrary rank can now be produced from the contact quadrics $\left(Q_{\zeta}, \alpha\right)$ considered earlier in this section. In the elliptic case, where $K$ is a subgroup of the centralizer $Z_{\mathbf{U}(n)}(\zeta)$, any choice of elements $t_{1}$, $t_{2}, \ldots, t_{k}$ of the centre $Z(K)$, which is a subgroup of the maximal torus $T$ of $\mathbf{U}(n)$, generates a strict contact action of $\Gamma$ on $Q_{\zeta}$ which commutes with $K$. In the hyperbolic case, where $K$ is a compact subgroup of $Z_{\mathbf{G L}(n, \mathbb{R}}(\zeta) /\langle\exp (\zeta)\rangle$, we may take any elements $h_{1}, h_{2}, \ldots, h_{k}$ of the split maximal torus $H$ of $\mathbf{G L}(n, \mathbb{R})$ which commute with $K$ to generate a strict contact action of $\Gamma$ on $Q_{\zeta}$ which commutes with $K$. In either case, we can then form the fattened mapping torus $\widehat{M}$, which is a conformal Hamiltonian $K$-manifold of Lee type with moment body the convex polyhedron $\Delta(\widehat{M})=\Delta\left(Q_{\zeta}\right)$.

\section{Appendix A. An instance of REAL SympleCtic CONVEXity}

The next theorem, which is a special case of a result from [34], is used in Section 7 (contact hyperboloids). Let $K$ be a compact connected Lie group with a choice of maximal torus $T$ and a closed chamber $C$ in $\mathfrak{t}^{*}$. The result concerns certain real algebraic subvarieties of a finite-dimensional unitary $K$-module $V$. The imaginary part of the Hermitian inner product $\langle\cdot, \cdot\rangle_{V}$ on $V$ is a symplectic form $\omega_{V}$, and a moment map for the $K$-action on $V$ is given by

$$
\Phi_{V}^{\xi}(v)=\frac{1}{2} \omega_{V}(\xi(v), v)=\frac{i}{2}\langle v, \xi(v)\rangle_{V}
$$

for $\xi \in \mathfrak{k}$ and $v \in V$. Let $W=V^{\mathbb{C}}=\mathbb{C} \otimes V$ be the complexified $K$-module, let $J$ be the complex structure of $V$, and let $W=V^{1,0} \oplus V^{0,1}$ be the usual eigenspace decomposition of $W$ respect to $J$. We have $V^{0,1}=\overline{V^{1,0}}$, where the conjugation is defined with respect to the totally real subspace $V$ of $W$. The map $f(v)=$ $\frac{1}{2}(v-i J v)$ is a complex linear isomorphism $V \cong V^{1,0}$. There is a unique $K$ module structure on $V^{1,0}$ that makes $f$ a $K$-module isomorphism. Likewise, the map $\bar{f}(v)=\frac{1}{2}(v+i J v)$ is a complex antilinear isomorphism $V \cong V^{0,1}$, and there is a unique $K$-module structure on $V^{0,1}$ that makes $\bar{f}$ an anti-isomorphism of $K$ modules. Thus $W=V^{1,0} \oplus V^{0,1}$ becomes a complex $K \times K$-module isomorphic to 
$V \oplus V^{*}$. We will write elements of $W$ as pairs $\left(f\left(v_{1}\right), \bar{f}\left(v_{2}\right)\right)$ with $v_{1}, v_{2} \in V$. In this notation complex conjugation is written as

$$
\left(f\left(v_{1}\right), \bar{f}\left(v_{2}\right)\right) \longmapsto\left(f\left(v_{2}\right), \bar{f}\left(v_{1}\right)\right) .
$$

The real inner product $\operatorname{Re}\langle\cdot, \cdot\rangle_{V}$ on $V$ extends to a Hermitian inner product $\langle\cdot, \cdot\rangle_{W}$ on $W$, and the symplectic form $\omega_{W}=\operatorname{Im}\langle\cdot, \cdot\rangle_{W}$ satisfies $f^{*} \omega_{W}=\frac{1}{2} \omega_{V}$ and $\bar{f}^{*} \omega_{W}=$ $-\frac{1}{2} \omega_{V}$. Therefore the moment map for the $K \times K$-action on $W$ is given by

$$
\Phi_{W}^{\left(\xi_{1}, \xi_{2}\right)}\left(f\left(v_{1}\right), \bar{f}\left(v_{2}\right)\right)=\frac{1}{2}\left(\Phi_{V}^{\xi_{1}}\left(v_{1}\right)-\Phi_{V}^{\xi_{2}}\left(v_{2}\right)\right) .
$$

A.2. Theorem. Let $V$ be a unitary $K$-module. Let $X$ be a $K$-invariant irreducible real algebraic subvariety of $V$. Let $X^{\mathbb{C}} \subseteq V^{\mathbb{C}}$ be the complexification of $X$. Suppose that $X^{\mathbb{C}}$ is $K \times K$-invariant and that $X$ contains at least one smooth point of $X^{\mathbb{C}}$. Then the moment body $\Delta(X)=\Phi_{V}(X) \cap C$ of $X$ is the convex hull of all dominant weights $\lambda \in C$ such that the $K \times K$-module with highest weight $(\lambda,-\lambda)$ occurs in the coordinate ring of $X^{\mathbb{C}}$. In particular $\Delta(X)$ is a rational convex polyhedral cone in $\mathfrak{k}^{*}$.

Proof. The strategy is to interpret $\mathfrak{k}$ as the tangent space to the symmetric space $(K \times K) / K$ and $X$ as a totally real Lagrangian in $X^{\mathbb{C}}$. Let $U=K \times K$ and $W=V^{\mathbb{C}}$. Define involutions $\sigma=\sigma_{U}$ of $U$ and $\sigma=\sigma_{W}$ of $W$ by $\sigma_{U}\left(k_{1}, k_{2}\right)=\left(k_{2}, k_{1}\right)$ and $\sigma_{W}(w)=\bar{w}$. Then $\sigma_{W}$ is antilinear and antisymplectic, and $X=\left(X^{\mathbb{C}}\right)^{\sigma_{W}}$, the set of $\sigma_{W}$-fixed points of $X$. Moreover,

$$
\begin{aligned}
\sigma_{W}\left(\left(k_{1}, k_{2}\right) \cdot\left(f\left(v_{1}\right), \bar{f}\left(v_{2}\right)\right)\right) & =\sigma_{W}\left(f\left(k_{1} v_{1}\right), \bar{f}\left(k_{2} v_{2}\right)\right) \\
& =\left(f\left(k_{2} v_{2}\right), \bar{f}\left(k_{1} v_{1}\right)\right) \\
& =\left(k_{2}, k_{1}\right) \cdot\left(f\left(v_{2}\right), \bar{f}\left(v_{1}\right)\right),
\end{aligned}
$$

which shows that $\sigma_{W}(u \cdot w)=\sigma_{U}(u) \cdot \sigma_{W}(w)$ for all $u \in U$ and $w \in W$. It follows that $\Phi_{W}\left(\sigma_{W}(w)\right)=-\sigma_{U}^{*}\left(\Phi_{W}(w)\right)$ for $w \in W$, where $\sigma_{U}^{*}$ is the transpose of the involution $\sigma_{U, *}$ of $\mathfrak{u}=\operatorname{Lie}(U)$ induced by $\sigma_{U}$. Let $\mathfrak{p}^{*}=\left\{\mu \in \mathfrak{u}^{*} \mid \sigma_{U}^{*}(\mu)=-\mu\right\}$. Then

$$
\mathfrak{p}^{*}=\left\{(\lambda,-\lambda) \mid \lambda \in \mathfrak{k}^{*}\right\} \cong \mathfrak{k}^{*},
$$

and the image $\Phi_{W}(X)$ is a subset of $\mathfrak{p}^{*}$. It follows from (A.1) that

$$
\Phi_{W}^{(\xi,-\xi)}(f(v), \bar{f}(v))=\frac{1}{2}\left(\Phi_{V}^{\xi}(v)-\Phi_{V}^{-\xi}(v)\right)=\Phi_{V}^{\xi}(v),
$$

so $\Phi_{V}=\left.\Phi_{W}\right|_{V}$ modulo the identification $\mathfrak{p}^{*} \cong \mathfrak{k}^{*}$. Hence $\Phi_{V}(X)=\Phi_{W}(X)$. The theorem now follows from [34, Theorem 6.3].

A.3. Example. Let $V=T^{*} \mathbb{R}^{n}=\mathbb{R}^{2 n}$ equipped with the standard symplectic structure $\omega$ and complex structure $J=\left(\begin{array}{cc}0 & -I \\ I & 0\end{array}\right)$. Then $V$ is a unitary $K$-module for any closed subgroup $K$ of $\mathbf{O}(n, \mathbb{R})$. Let $\zeta=\operatorname{diag}\left(\zeta_{1}, \zeta_{2}, \ldots, \zeta_{n}\right)$, where $\zeta_{1} \geq \zeta_{2} \geq$ $\cdots \geq \zeta_{n}>0$ and suppose that $K$ centralizes $\zeta$. Then the action of $K$ preserves the hyperboloid

$$
X=\left\{(x, y) \in V \mid x^{\top} \zeta y=1\right\} .
$$

To complexify the hyperboloid we substitute new variables $x=\frac{1}{2}(z+\bar{z})$ and $y=$ $\frac{1}{2 i}(z-\bar{z})$ and obtain

$$
X^{\mathbb{C}}=\left\{(z, \bar{z}) \in V^{1,0} \oplus V^{0,1} \mid z^{\top} \zeta z-\bar{z}^{\top} \zeta \bar{z}=4 i\right\} .
$$


Evidently $X^{\mathbb{C}}$ is unchanged by substitutions of the form $z \mapsto k_{1} z, \bar{z} \mapsto k_{2} \bar{z}$ with $k_{1}$, $k_{2} \in K$. The variables $z$ are coordinates on $V^{1,0}$ and the variables $\bar{z}$ are coordinates on $V^{0,1}$, so we see that $X^{\mathbb{C}}$ is preserved by the action of $K \times K$. It now follows from Theorem A.2 that the moment body $\Delta(X)$ of $X$ with respect to the $K$-action is a rational convex polyhedral cone.

A.4. Example. Let $V=\mathbb{C}^{n}$ equipped with the standard unitary structure. Then $V$ is a unitary $K$-module for any closed subgroup $K$ of $\mathbf{U}(n)$. Let $\zeta$ be as in Example A.3 and suppose that $K$ centralizes $\zeta$. Then $K$ acts on the ellipsoid

$$
X=\left\{z \in V \mid z^{*} \zeta z=1\right\} .
$$

The complexified ellipsoid is

$$
X^{\mathbb{C}}=\left\{(z, \bar{z}) \in V^{1,0} \oplus V^{0,1} \mid \bar{z}^{\top} \zeta z=1\right\},
$$

which is not preserved by the action of $K \times K$, so Theorem A.2 does not apply. Obviously the moment body $\Delta(X)$ cannot be a cone because $X$ is compact. However, the contact manifold $X$ is the level set of the $\zeta$-component of the $\mathbf{U}(n)$-action on $\mathbb{C}^{n}$, so it follows from the contact convexity theorem [30, Theorem 4.5.1] that $\Delta(X)$ is a (not necessarily rational) convex polytope.

\section{Appendix B. Conformal PResymplectic CONVEXity}

The conformal symplectic convexity theorem, Theorem 1.1, extends without difficulty to conformal presymplectic manifolds, where the 2-form is of constant rank less than the dimension. The purpose of this appendix is to briefly explain this generalization, which uses the techniques of [30].

Transverse vector fields and basic differential forms. Let $\mathcal{F}$ be a foliation of a manifold $P$ and $\mathfrak{X}(\mathcal{F})$ the Lie algebra of vector fields tangent to $\mathcal{F}$. Recall from Section 5 that the Lie algebra of foliate vector fields is the normalizer $N_{\mathfrak{X}(P)}(\mathfrak{X}(\mathcal{F}))$ of $\mathfrak{X}(\mathcal{F})$ inside the Lie algebra $\mathfrak{X}(P)$ of all vector fields. The quotient Lie algebra

$$
\mathfrak{X}(P, \mathcal{F})=N_{\mathfrak{X}(P)}(\mathfrak{X}(\mathcal{F})) / \mathfrak{X}(\mathcal{F})
$$

is the Lie algebra of transverse vector fields of the foliation. A transverse vector field $X$ is not a vector field, but an equivalence class of vector fields. The flow of $X$ maps leaves of $\mathcal{F}$ to leaves of $\mathcal{F}$, but is well-defined only up to a flow along the leaves of $\mathcal{F}$. On every transversal of the foliation $X$ induces a genuine vector field. (Hence $\mathfrak{X}(M, \mathcal{F})=\mathfrak{X}(M / \mathcal{F})$ if the leaf space $M / \mathcal{F}$ is a manifold.)

A differential form $\alpha \in \Omega^{*}(M)$ is $\mathcal{F}$-basic if $\iota(X) \alpha=\iota(X) d \alpha=0$ for all $X \in$ $\mathfrak{X}(\mathcal{F})$. We denote the complex of $\mathcal{F}$-basic forms by $\Omega^{*}(M, \mathcal{F})$ and the algebra of $\mathcal{F}$-basic functions by $\mathcal{C}^{\infty}(M, \mathcal{F})=\Omega^{0}(M, \mathcal{F})$.

Conformal presymplectic forms. A conformal presymplectic structure on a manifold $M$ is a pair $(\omega, \theta)$, where $\omega$ is a 2 -form of constant rank and $\theta$ a closed 1-form satisfying $d_{\theta} \omega=0$ and $\iota(X) \theta=0$ for all vector fields $X$ with $\iota(X) \omega=0$. As in the nondegenerate case we call $\theta$ the Lee form and its cohomology class the Lee class, and we say $(\omega, \theta)$ is strict if $\theta$ is not exact, and global if $\theta$ is exact.

Let $(M, \omega, \theta)$ be a conformal presymplectic manifold. The kernel of $\omega$ is an involutive subbundle of $T M$, which generates a (regular) foliation $\mathcal{F}=\mathcal{F}_{\omega}$ called the null foliation of $\omega$. The forms $\omega$ and $\theta$ are basic with respect to this foliation, and $(\omega, \theta)$ induces a conformal symplectic structure on every transversal of the 
foliation. In this sense a conformal presymplectic structure is a transverse analogue of a conformal symplectic structure.

Suppose a function $f \in \mathcal{C}^{\infty}(M)$ and a vector field $X \in \mathfrak{X}(M)$ satisfy $d_{\theta} f=$ $\iota(X) \omega$. Then $f$ must be $\mathcal{F}$-basic, and $X$ is determined by $f$ only up to an element of $\mathfrak{X}(\mathcal{F})$. Moreover, $L_{\theta}(X) \omega=0$, so

$$
\omega([X, Y], Z)=-\omega(Y,[X, Z])-\theta(X) \omega(Y, Z)
$$

for all $Y, Z \in \mathfrak{X}(M)$. This shows that $[X, Y] \in \mathfrak{X}(\mathcal{F})$ for all $Y \in \mathfrak{X}(\mathcal{F})$; in other words $X$ is foliate and therefore represents a transverse vector field $X_{f} \in \mathfrak{X}(M, \mathcal{F})$, called the Hamiltonian vector field of $f$. Thus the Hamiltonian correspondence $f \mapsto X_{f}$ is a map

$$
\mathcal{C}^{\infty}(M, \mathcal{F}) \longrightarrow \mathfrak{X}(M, \mathcal{F}) .
$$

In particular the Lee vector field is the transverse vector field $A \in \mathfrak{X}(M, \mathcal{F})$ defined by $A=X_{1}$.

Suppose $K$ acts on $M$ by foliate transformations. Then the infinitesimal action $\mathfrak{k} \rightarrow \mathfrak{X}(M)$ takes values in $N_{\mathfrak{X}(\mathcal{F})}(\mathfrak{X}(M))$. Composing this map with the quotient map gives a homomorphism $\mathfrak{k} \rightarrow \mathfrak{X}(M, \mathcal{F})$. As in the nondegenerate case, we say the action is weakly Hamiltonian if there is a linear lifting $\Phi^{\vee}$ of this homomorphism,

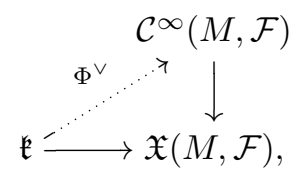

and the map $\Phi: M \rightarrow \mathfrak{k}^{*}$ dual to $\Phi^{\vee}$ is the moment map. The $K$-action is Hamiltonian, or $M$ is a Hamiltonian conformal presymplectic $K$-manifold, if there exists a moment map $\Phi$ which is $K$-equivariant. The action is of Lee type if the transverse Lee vector field $A$ has a foliate representative of the form $\zeta_{M}$ with $\zeta \in \mathfrak{k}$. We can now state the conformal presymplectic convexity theorem.

B.1. Theorem. Let $K$ be a compact connected Lie group which acts on a connected strict conformal presymplectic manifold $(M, \omega, \theta)$ in a Hamiltonian fashion. Assume that the $K$-action is clean and of Lee type, and that the moment map $\Phi: M \rightarrow \mathfrak{k}^{*}$ is proper. Choose a maximal torus $T$ of $K$ and a closed Weyl chamber $C$ in $\mathfrak{t}^{*}$, where $\mathfrak{t}=\operatorname{Lie}(T)$.

(i) The fibres of $\Phi$ are connected and $\Phi: M \rightarrow \Phi(M)$ is an open map.

(ii) $\Delta(M)=\Phi(M) \cap C$ is a closed convex polyhedral set.

Outline of proof. The essential fact that $d_{\theta}: \mathcal{C}^{\infty}(M) \rightarrow \Omega^{1}(M)$ is injective (Proposition 2.4) remains valid for conformal presymplectic manifolds, as is clear from the proof in [38]. None of the auxiliary results in Section 3 uses the nondegeneracy of $\omega$. The tubular neighbourhood theorem, Proposition 3.4, generalizes as follows: for every $m \in M$ and every equivariant tubular neighborhood $V$ of $K \cdot m$ there exists a $K$-invariant smooth function $f$ on $V$ such that $\left.\theta\right|_{V}=d f$; the form $\Omega=\left.e^{f} \cdot \omega\right|_{V}$ is presymplectic, the $K$-action on $(V, \Omega)$ is Hamiltonian with equivariant moment map $\Psi=\left.e^{f} \cdot \Phi\right|_{V}$, and the null foliation of $\Omega$ is $\mathcal{F}_{\Omega}=\left.\mathcal{F}_{\omega}\right|_{V}$. In particular, the $K$-action on $V$ is clean with respect to the foliation $\mathcal{F}_{\Omega}$. In the proof of the local convexity theorem, Theorem 4.3, instead of appealing to the symplectic version of the local convexity theorem, we need to appeal to the presymplectic local convexity theorem, [30, Theorem 2.12.1]. The remainder of the proof, and the proof of Theorem 1.1, then go through unchanged. 


\section{REFERENCES}

1. M. F. Atiyah, Convexity and commuting Hamiltonians, Bull. London Math. Soc. 14 (1982), no. 1, 1-15. MR 6424161

2. G. Bande and D. Kotschick, Contact pairs and locally conformally symplectic structures, Harmonic maps and differential geometry (E. Loubeau and S. Montaldo, eds.), Contemp. Math., vol. 542, Amer. Math. Soc., Providence, RI, 2011, pp. 85-98. MR 27966422

3. G. Bazzoni and J. C. Marrero, On locally conformal symplectic manifolds of the first kind, Bull. Sci. Math. 143 (2018), 1-57. MR 376742619

4. F. Belgun, On the metric structure of non-Kähler complex surfaces, Math. Ann. 317 (2000), no. 1, 1-40. MR 17606672

5. F. Belgun, O. Goertsches, and D. Petrecca, Locally conformally symplectic convexity, J. Geom. Phys. 135 (2019), 235-252. MR 3872931 2, 5, 6, 7, 12, 13, 14, 15

6. B. Chantraine and E. Murphy, Conformal symplectic geometry of cotangent bundles, Tech. report, to appear, arXiv:1606.00861.2

7. R. Chiang and Y. Karshon, Convexity package for momentum maps on contact manifolds, Algebr. Geom. Topol. 10 (2010), no. 2, 925-977. MR 26297712

8. P. Dazord, A. Lichnerowicz, and C.-M. Marle, Structure locale des variétés de Jacobi, J. Math. Pures Appl. (9) 70 (1991), no. 1, 101-152. MR 10919225

9. Y. Eliashberg and E. Murphy, Making cobordisms symplectic, eprint, 2015, arXiv:1504.06312. 2,4

10. M. Farber, Topology of closed one-forms, Mathematical Surveys and Monographs, vol. 108, American Mathematical Society, Providence, RI, 2004. MR 203460118

11. A. Gray and L. M. Hervella, The sixteen classes of almost Hermitian manifolds and their linear invariants, Ann. Mat. Pura Appl. (4) 123 (1980), 35-58. MR 5819242

12. F. Guedira and A. Lichnerowicz, Géométrie des algèbres de Lie locales de Kirillov, J. Math. Pures Appl. (9) 63 (1984), no. 4, 407-484. MR 789560 2, 5

13. V. Guillemin, V. Ginzburg, and Y. Karshon, Moment maps, cobordisms, and Hamiltonian group actions, Mathematical Surveys and Monographs, vol. 98, American Mathematical Society, Providence, RI, 2002, Appendix J by M. Braverman. 7

14. V. Guillemin, E. Miranda, A. R. Pires, and G. Scott, Convexity for Hamiltonian torus actions on b-symplectic manifolds, Math. Res. Lett. 24 (2017), no. 2, 363-377. MR 36852752

15. V. Guillemin and S. Sternberg, Convexity properties of the moment mapping, Invent. Math. 67 (1982), no. 3, 491-513. MR 6641171

16. S. Haller and T. Rybicki, Reduction for locally conformal symplectic manifolds, J. Geom. Phys. 37 (2001), no. 3, 262-271. MR 1807280 5, 6

17. J. Hilgert, K.-H. Neeb, and W. Plank, Symplectic convexity theorems and coadjoint orbits, Compositio Math. 94 (1994), no. 2, 129-180. MR 1302314 10, 11

18. B. Hoffman and R. Sjamaar, Stacky hamiltonian actions and symplectic reduction, eprint, 2018, with an appendix by C. Zhu, arXiv:1808.01003. 26

19. H. Imanishi, On codimension one foliations defined by closed one-forms with singularities, J. Math. Kyoto Univ. 19 (1979), no. 2, 285-291. MR 54570918

20. F. Kirwan, Convexity properties of the moment mapping, III, Invent. Math. 77 (1984), no. 3, 547-552. MR 759257 2, 11

21. B. Kostant, On convexity, the Weyl group and the Iwasawa decomposition, Ann. Sci. École Norm. Sup. (4) 6 (1973), 413-455 (1974). MR 03645521

22. J. L. Koszul, Sur certains groupes de transformations de Lie, Géométrie différentielle (Strasbourg, 1953), Colloques internationaux du Centre national de la Recherche scientifique, 1953, pp. $137-141.7$

23. H.-C. Lee, A kind of even-dimensional differential geometry and its application to exterior calculus, Amer. J. Math. 65 (1943), 433-438. MR 00084952

24. J. Lefebvre, Propriétés du groupe des transformations conformes et du groupe des automorphismes d'une variété localement conformément symplectique, C. R. Acad. Sci. Paris Sér. A-B 268 (1969), A717-A719. MR 02448862

25. E. Lerman, A convexity theorem for torus actions on contact manifolds, Illinois J. Math. 46 (2002), no. 1, 171-184. MR 19360832

26. E. Lerman, E. Meinrenken, S. Tolman, and C. Woodward, Nonabelian convexity by symplectic cuts, Topology 37 (1998), no. 2, 245-259. MR 148920314 
27. E. Lerman and S. Tolman, Hamiltonian torus actions on symplectic orbifolds and toric varieties, Trans. Amer. Math. Soc. 349 (1997), no. 10, 4201-4230. MR 14015252

28. P. Libermann, Sur les structures presque complexes et autres structures infinitésimales régulières, Bull. Soc. Math. France 83 (1955), 195-224. MR 00797662

29. A. Lichnerowicz, Les variétés de Jacobi et leurs algèbres de Lie associées, J. Math. Pures Appl. (9) 57 (1978), no. 4, 453-488. MR 5246295

30. Y. Lin and R. Sjamaar, Convexity properties of presymplectic moment maps, J. Symplectic Geom., to appear, arXiv:1706.00520. 2, 11, 12, 18, 21, 28, 29

31. I. Moerdijk and J. Mrčun, Introduction to foliations and Lie groupoids, Cambridge Studies in Advanced Mathematics, vol. 91, Cambridge University Press, Cambridge, 2003. MR 2012261 16

32. Y. Nitta, Convexity properties of generalized moment maps, J. Math. Soc. Japan 61 (2009), no. 4, 1171-1204. MR 25885082

33. L. Ornea, M. Verbitsky, and V. Vuletescu, Classification of non-Kähler surfaces and locally conformally Kähler geometry, eprint, 2018, arXiv:1810.05768. 2

34. L. O'Shea and R. Sjamaar, Moment maps and Riemannian symmetric pairs, Math. Ann. 317 (2000), no. 3, 415-457. MR 1776111 26, 27

35. T. Ratiu and N. T. Zung, Presymplectic convexity and (ir)rational polytopes, eprint, 2017, arXiv:1705.11110. 2

36. R. Sjamaar, Convexity properties of the moment mapping re-examined, Adv. Math. 138 (1998), no. 1, 46-91. MR 1645052 9, 22, 23

37. R. Sjamaar and E. Lerman, Stratified symplectic spaces and reduction, Ann. of Math. (2) 134 (1991), no. 2, 375-422. MR 112747915

38. I. Vaisman, Locally conformal symplectic manifolds, Internat. J. Math. Math. Sci. 8 (1985), no. 3, 521-536. MR 809073 2, 5, 19, 29

39. M. P. Wojtkowski and C. Liverani, Conformally symplectic dynamics and symmetry of the Lyapunov spectrum, Comm. Math. Phys. 194 (1998), no. 1, 47-60. MR 16282862

Y. Chen, School of Science, Chongqing University of Technology, Chongqing 400054, CHINA

Current address: Department of Mathematics, Pennsylvania State University, USA

E-mail address: youmingchen@cqut.edu.cn

R. Sjamaar, Department of Mathematics, Cornell University, Ithaca, NY 14853-4201, USA

E-mail address: sjamaar@math.cornell.edu

X. Yang, Department of Mathematics, Chongqing University, Chongqing 401331, CHINA

Current address: Department of Mathematics, Cornell University, Ithaca, NY 14853-4201, USA

E-mail address: xy373@cornell.edu 